\title{
TrAJECTORIAL DiSSIPATION AND GRADIENT FLOW FOR THE RELATIVE ENTROPY IN MARKOV CHAINS *
}

\author{
IOANNIS KARATZAS ${ }^{\dagger}$ JAN MAAS ${ }^{\ddagger} \quad$ WALTER SCHACHERMAYER ${ }^{\S}$
}

May 19, 2022

\begin{abstract}
We study the temporal dissipation of variance and relative entropy for ergodic Markov Chains in continuous time, and compute explicitly the corresponding dissipation rates. These are identified, as is well known, in the case of the variance in terms of an appropriate Hilbertian norm; and in the case of the relative entropy, in terms of a Dirichlet form which morphs into a version of the familiar Fisher information under conditions of detailed balance. Here we obtain trajectorial versions of these results, valid along almost every path of the random motion and most transparent in the backwards direction of time. Martingale arguments and time reversal play crucial roles, as in the recent work of Karatzas, Schachermayer and Tschiderer for conservative diffusions. Extensions are developed to general "convex divergences" and to countable state-spaces. The steepest descent and gradient flow properties for the variance, the relative entropy, and appropriate generalizations, are studied along with their respective geometries under conditions of detailed balance, leading to a very direct proof for the HWI inequality of Otto and Villani in the present context.
\end{abstract}

Keywords and Phrases: Markov Chain; Relative Entropy; Time Reversal; Steepest Descent; Gradient Flow. AMS 2020 Subject Classifications: 60J27; 60H10; 60G44; 46C05.

\section{Introduction and Summary}

We present a trajectorial approach to the temporal dissipation of variance and relative entropy, in the context of ergodic MARKOV Chains in continuous time. We follow the methodology of the recent work by KARATZAS, SCHACHERMAYER \& TSCHIDERER (2020), which is based on stochastic calculus and uses time-reversal in a critical fashion. By aggregating the trajectorial results, i.e., by averaging them with respect to the invariant measure, we obtain a very crisp, geometric picture of the steepest descent property for the curve of time-marginals, relative to local perturbations. This holds for an appropriate, locally flat metric on configuration space, defined in terms of a suitable discrete SOBOLEV norm.

${ }^{*}$ We are indebted to Ioannis Kontoyiannis, Peter Michor, Abishek Tilva and Lane Yeung for sharing their expertise on the subject, for bringing relevant literature to our attention, and for their many helpful comments. I.K. acknowledges support from the U.S. National Science Foundation under Grant NSF-DMS-20-04997. J.M. acknowledges support from the European Research Council (ERC) under the European Union's Horizon 2020 research and innovation programme (grant agreement No 716117) and from the Austrian Science Fund (FWF) through project F65. W.S. acknowledges support from the Austrian Science Fund (FWF) under grant P28861 and by the Vienna Science and Technology Fund (WWTF) through projects MA14-008 and MA16-021.

$\dagger$ Department of Mathematics, Columbia University, 2990 Broadway, New York, NY 10027, USA (ik1@ columbia.edu), and INTECH Investment Management, One Palmer Square, Suite 441, Princeton, NJ 08542, USA (ikaratzas@intechjanus.com).

$¥$ Institute of Science and Technology (IST) Austria, Am Campus 1, 3400 Klosterneuburg, Austria (email: jan.maas@ist.ac.at).

$\S$ Faculty of Mathematics, University of Vienna, Oskar-Morgenstern-Platz 1, 1090 Vienna, Austria (email: walter.schachermayer@univie.ac.at). 
We adopt then a more global approach, and establish also the gradient flow property-to the effect that the temporal evolution for the curve of the Chain's time-marginals is prescribed by an appropriate Riemannian metric on the manifold of probability measures on configuration space, and by the differential of the relative entropy functional along this curve; cf. MAAS (2011), MIELKE (2011), ERBAR \& MAAS (2012, 2014). Both steepest descent and gradient flow are manifestations of the seminal JORDAN, KINDERLEHRER \& Отто (1998) results and of their outgrowth, the so-called "Oтто Calculus" initiated in Отто (2001).

Preview: For a finite state-space, we set up the probabilistic framework in Section 2 and the functionalanalytic one in Section 4. The appropriate stochastic-analytic machinery and results appear in Sections 3 and 5. Temporal dissipation and steepest descent are developed in increasing generality: First in Section 6 for the variance and its associated, globally determined and flat, metric; then in Section 7 for the BOLTZMANN-GIBBS-SHANNON relative entropy; and finally in Section 8 for general entropies induced by convex functions. Gradient flows and their associated geometries are taken up in Section 9, culminating with a very direct proof of a discrete version of the celebrated HWI inequality of OTTO \& VILLANI (2000). Some extensions to state-spaces with a countable infinity of elements are developed in Section 10.

\section{The Setting}

On a probability space $(\Omega, \mathcal{F}, \mathbb{P})$, we start with an irreducible, positive recurrent, discrete-time MARKOV Chain $\mathcal{Z}=\left(Z_{n}\right)_{n \in \mathbb{N}_{0}}$ with state-space $\mathcal{S}$, transition probability matrix $\Pi=\left(\pi_{x y}\right)_{(x, y) \in \mathcal{S}^{2}}$ with entries $\pi_{x y}=\mathbb{P}\left(Z_{n+1}=y \mid Z_{n}=x\right)$ for $n \in \mathbb{N}_{0}$, and initial distribution $P(0)=(p(0, x))_{x \in \mathcal{S}}$ which is a column vector with components $p(0, x):=\mathbb{P}\left(Z_{0}=x\right)>0$ for all $x \in \mathcal{S}$. Throughout Sections 2-9, the state-space $\mathcal{S}$ is assumed to be finite; extensions to countable state-spaces are taken up in Section 10.

It is straightforward to check that the sequence of random variables $\left(M_{n}^{f}\right)_{n \in \mathbb{N}_{0}}$ with $M_{0}^{f}:=f\left(Z_{0}\right)$,

$$
M_{n}^{f}:=f\left(Z_{n}\right)-\sum_{k=0}^{n-1}(\Pi f-f)\left(Z_{k}\right), \quad n \in \mathbb{N},
$$

is a martingale of the filtration generated by the MARKOV Chain $\mathcal{Z}$, for any given function $f: \mathcal{S} \rightarrow \mathbb{R}$. Here and in what follows, we denote $(\Pi f)(z):=\sum_{y \in \mathcal{S}} \pi_{z y} f(y), z \in \mathcal{S}$.

It is well known that such a Chain has a unique invariant distribution: that is, a column vector $Q=$ $(q(y))_{y \in \mathcal{S}}$ of positive numbers adding up to 1 and satisfying $\Pi^{\prime} Q=Q$ or, more explicitly,

$$
q(y)=\sum_{z \in \mathcal{S}} q(z) \pi_{z y}, \quad \forall y \in \mathcal{S} .
$$

Here and throughout this paper, prime' denotes transposition of a matrix or vector. A major result of discrete-time MARKOV Chain theory states that, when $\mathcal{Z}$ is also aperiodic, the $k$-step transition probabilities

$$
\pi_{x y}^{(0)}:=\mathbf{1}_{x=y}, \quad \pi_{x y}^{(k)}:=\mathbb{P}\left(Z_{k}=y \mid Z_{0}=x\right), \quad k \in \mathbb{N}
$$

converge as $k$ tends to infinity to $q(y)$, for every pair of states $(x, y) \in \mathcal{S}^{2}$. We refer to Chapter 1 in NORRIS (1997), in particular Theorems 1.7.7 and 1.8.3, for an excellent account of the relevant theory.

\subsection{From Discrete- to Continuous-Time Markov Chains, via PoIsson}

Consider now on the same probability space a POISSON process $\mathcal{N}=(N(t))_{0 \leq t<\infty}$ with parameter $\lambda=1$ and independent of the discrete-time MARKOV Chain $\mathcal{Z}$. We construct via time-change the continuous-time process

$$
X(t):=Z_{N(t)}, \quad 0 \leq t<\infty
$$


as well as the filtration $\mathbb{F}^{X}=\left\{\mathcal{F}^{X}(t)\right\}_{0 \leq t<\infty}$ this process generates via $\mathcal{F}^{X}(t):=\sigma(X(s), 0 \leq s \leq t)$. Straightforward computation shows that this new, continuous-time process $\mathcal{X}=(X(t))_{0 \leq t<\infty}$ has the MARKOV property, and time-homogeneous transition probabilities

$$
\varrho_{h}(x, y):=\mathbb{P}(X(t+h)=y \mid X(t)=x)=e^{-h} \sum_{k \in \mathbb{N}_{0}} \frac{h^{k}}{k !} \pi_{x y}^{(k)}, \quad t \geq 0, h>0
$$

with the notation of (2.3); we set $\varrho_{0}(x, y):=\mathbf{1}_{x=y}$. The functions $h \mapsto \varrho_{h}(x, y)$ in (2.5) are uniformly continuous and continuously differentiable; cf. Theorems 2.13, 2.14 in LigGETT (2010).

More generally, for arbitrary $n \in \mathbb{N}, 0<\theta_{1}<\cdots<\theta_{n}=\theta<t<\infty,\left(x, y_{1}, \cdots, y_{n}, z\right) \in \mathcal{S}^{n+2}$ with $y=y_{n}$, the finite-dimensional distributions of this process are

$$
\begin{aligned}
\mathbb{P}(X(0) & \left.=x, X\left(\theta_{1}\right)=y_{1}, \cdots, X\left(\theta_{n}\right)=y_{n}, X(t)=z\right)= \\
& =p(0, x) \varrho_{\theta_{1}}\left(x, y_{1}\right) \varrho_{\theta_{2}-\theta_{1}}\left(y_{1}, y_{2}\right) \cdots \varrho_{\theta_{n}-\theta_{n-1}}\left(y_{n-1}, y_{n}\right) \cdot \varrho_{t-\theta}(y, z)
\end{aligned}
$$

and we deduce the time-homogeneous MARKOV property

$$
\mathbb{P}\left(X(t)=z \mid \mathcal{F}^{X}(\theta)\right)=\varrho_{t-\theta}(X(\theta), z)=\mathbb{P}(X(t)=z \mid X(\theta)) .
$$

Finally, from the CHAPMAN-Kolmogorov equations $\pi_{x y}^{(m+n)}=\sum_{z \in \mathcal{S}} \pi_{x z}^{(m)} \pi_{z y}^{(n)}$ for the $k$-step transition probabilities of $\mathcal{Z}$ in (2.3), we deduce these same equations for the quantities in (2.5):

$$
\varrho_{t+\theta}(x, y)=\sum_{z \in \mathcal{S}} \varrho_{\theta}(x, z) \varrho_{t}(z, y), \quad(\theta, t) \in[0, \infty)^{2}, \quad(x, y) \in \mathcal{S}^{2}
$$

Here we think of the temporal argument $\theta$ as the "backward variable", and of $t$ as the "forward variable".

\subsection{Infinitesimal Generators and Martingales}

We introduce now the matrix

$$
\mathcal{K}:=\Pi-\mathrm{I}=\{\kappa(x, y)\}_{(x, y) \in \mathcal{S}^{2}} \quad \text { with elements } \quad \kappa(x, y):=\pi_{x y}-\mathbf{1}_{x=y}:
$$

non-negative off the diagonal, adding up to zero across each row. From (2.5) and with the help of timehomogeneity, we obtain for $t \geq 0, h>0$ the infinitesimal "transition rates"

$$
\begin{gathered}
\mathbb{P}(X(t+h)=y \mid X(t)=x)=h \cdot \kappa(x, y)+o(h), \quad x \neq y, \\
\mathbb{P}(X(t+h)=x \mid X(t)=x)=1+h \cdot \kappa(x, x)+o(h)
\end{gathered}
$$

with the standard convention $\lim _{h \downarrow 0}(o(h) / h)=0$, valid uniformly over $t \in[0, \infty)$. In particular, (2.10) and (2.11) give the infinitesimals $\varrho_{h}(x, y)-\varrho_{0}(x, y)=h \cdot \kappa(x, y)+o(h)$ for all $(x, y) \in \mathcal{S}^{2}$, and thus

$$
\left.\partial \varrho_{h}(x, y)\right|_{h=0}=\kappa(x, y) .
$$

Here and throughout the paper, $\partial g$ denotes partial differentiation of a function $g$ with respect to its temporal argument.

A bit more generally, for any $f: \mathcal{S} \rightarrow \mathbb{R}$ we have from (2.10), (2.11) the semigroup computation

$$
\left(T_{h} f\right)(x):=\mathbb{E}[f(X(t+h)) \mid X(t)=x]=f(x)+h \cdot(\mathcal{K} f)(x)+o(h) .
$$

We deploy, here and in what follows, the infinitesimal generator of the Chain, i.e., the linear operator

$$
(\mathcal{K} f)(x):=(\Pi f)(x)-f(x)=\sum_{y \in \mathcal{S}} \kappa(x, y) f(y)=\sum_{y \in \mathcal{S}} \kappa(x, y)[f(y)-f(x)], \quad x \in \mathcal{S} .
$$


Using the computation (2.13), it is shown fairly easily that the exact analogue of the random sequence (2.1) in our present setting, namely, the process

$$
f(X(t))-\int_{0}^{t}(\mathcal{K} f)(X(\theta)) \mathrm{d} \theta, \quad 0 \leq t<\infty
$$

is an $\mathbb{F}^{X}$-martingale; cf. Theorem 3.32 in LiggetT (2010). As a slight generalization, we obtain also the following result (Lemma IV.20.12 in ROGERS \& WILLIAMS (1987)).

Proposition 2.1. Given any function $g:[0, \infty) \times \mathcal{S} \rightarrow \mathbb{R}$ whose temporal derivative $t \mapsto \partial g(t, x)$ is continuous for every state $x \in \mathcal{S}$, the process below is a local $\mathbb{F}^{X}$-martingale:

$$
M^{g}(t):=g(t, X(t))-\int_{0}^{t}(\partial g+\mathcal{K} g)(\theta, X(\theta)) \mathrm{d} \theta, \quad 0 \leq t<\infty .
$$

Remark 2.1. The General Case: Instead of starting with transition probabilities $\pi_{x y}$ and defining $\kappa(x, y)=$ $\pi_{x y}-\mathbf{1}_{x=y}$ as in (2.9), one can work instead with any transition rates $\kappa(x, y)$ satisfying: $(i) \kappa(x, y) \geq 0$ for $x \neq y$; and $(i) \sum_{y \in \mathcal{S}} \kappa(x, y)=0$ for every $x \in \mathcal{S}$. In this manner, arbitrary irreducible continuous-time MARKOV chains on finite state spaces can be constructed, and studied with little extra effort. We have opted here for the somewhat less general, but very concrete and intuitive, approach of the present Section.

\section{Forward and Backward KolMogorov Equations}

Let us differentiate both sides of the equations in (2.8) with respect to the backward variable $\theta$, then set $\theta=0$. We obtain on account of (2.12) the Backward KOLMOGOROV differential equations

$$
\partial \varrho_{t}(x, y)=\sum_{z \in \mathcal{S}} \kappa(x, z) \varrho_{t}(z, y)
$$

We can write this system of equations, for the matrix-valued function $t \mapsto \mathcal{P}_{t}=\left(\varrho_{t}(x, y)\right)_{(x, y) \in \mathcal{S}^{2}}$ of the forward variable $t \in[0, \infty)$, in the form $\partial \mathcal{P}_{t}=\mathcal{K} \mathcal{P}_{t}, \mathcal{P}_{0}=\mathrm{I}$.

In a similar manner, differentiating formally the equations (2.8) with respect to the forward variable $t$, then evaluating at $t=0$ and recalling the transpose

$$
\mathcal{K}^{\prime}:=\left(\kappa^{\prime}(y, z)\right)_{(y, z) \in \mathcal{S}^{2}}, \quad \kappa^{\prime}(y, z):=\kappa(z, y)
$$

of the $\mathcal{K}$-matrix, we obtain the Forward Kolmogorov equations

$$
\partial \varrho_{\theta}(x, y)=\sum_{z \in \mathcal{S}} \varrho_{\theta}(x, z) \kappa(z, y)=\sum_{z \in \mathcal{S}} \kappa^{\prime}(y, z) \varrho_{\theta}(x, z), \quad \text { or } \quad \partial \mathcal{P}_{\theta}=\mathcal{K}^{\prime} \mathcal{P}_{\theta}, \quad \mathcal{P}_{0}=\mathrm{I} .
$$

\subsection{A Curve of Probability Vectors}

For every $t>0$, let us consider the column vector $P(t)=(p(t, y))_{y \in \mathcal{S}}$ of probabilities for the $\mathbb{P}$-distribution

$$
p(t, y):=\mathbb{P}(X(t)=y)=e^{-t} \sum_{x \in \mathcal{S}} p(0, x) \sum_{k \in \mathbb{N}_{0}} \frac{t^{k}}{k !} \pi_{x y}^{(k)}>0
$$

of the random variable $X(t)$. The forward Kolmogorov equations of (3.3), the law of total probability, and the MARKOV property, show that these satisfy their own forward KOLMOGOROV equations, namely

$$
\partial p(t, y)=\sum_{z \in \mathcal{S}} p(t, z) \kappa(z, y)=\sum_{z \in \mathcal{S}} \kappa^{\prime}(y, z) p(t, z)=:\left(\mathcal{K}^{\prime} p\right)(t, y)
$$


or, more compactly and in matrix form, $\partial P(t)=\mathcal{K}^{\prime} P(t), \quad 0 \leq t<\infty$ in the notation of (3.2). We shall think of $(P(t))_{0 \leq t<\infty}$ as a curve on the manifold $\mathcal{M}=\mathcal{P}_{+}(\mathcal{S})$, of vectors $P=(p(x))_{x \in \mathcal{S}}$ with strictly positive elements and total mass $\sum_{x \in \mathcal{S}} p(x)=1$, viewed as probability measures and governed by (3.5).

Suppose that the initial distribution $P(0)$ of the discrete-time MARKOV Chain $\mathcal{Z}$ coincides with the column vector $Q=(q(y))_{y \in \mathcal{S}}$ of (2.2) satisfying $\Pi^{\prime} Q=Q$, or equivalently $\mathcal{K}^{\prime} Q=0$ on account of (2.9). It follows that $P(t) \equiv Q, \forall t \in[0, \infty)$ provides now the solution of (3.5): the distribution $Q$ is invariant also for the continuous-time MARKOV Chain $\mathcal{X}$ in (2.4).

A bit more generally, $Q$ is the equilibrium distribution of $\mathcal{X}$, in the sense that for every initial distribution $P(0)=(p(0, x))_{x \in \mathcal{S}}$ and function $f: \mathcal{S} \rightarrow \mathbb{R}$ we have the limiting behavior

$$
\begin{gathered}
\lim _{t \rightarrow \infty} p(t, y)=q(y), \quad \forall y \in \mathcal{S}, \\
\lim _{T \rightarrow \infty} \frac{1}{T} \int_{0}^{T} f(X(t)) \mathrm{d} t=\sum_{y \in \mathcal{S}} q(y) f(y), \quad \mathbb{P} \text { - a.e.; }
\end{gathered}
$$

see Sections 3.6-3.8 in NORRIS (1997) for an account of these results. In the present, continuous-time context, aperiodicity plays no role.

\subsection{A Curve of Likelihood Ratios}

Let us compare now the components of the probability vector $P(t)$ in (3.4), with those of the invariant probability vector $Q$ in (2.2). One way to do this, very fruitful in the present context, is by considering the likelihood ratio column vector

$$
\ell_{t} \equiv \ell(t)=(\ell(t, y))_{y \in \mathcal{S}} \quad \text { with components } \quad \ell(t, y):=\frac{p(t, y)}{q(y)} .
$$

Substituting the product $p(t, y)=\ell(t, y) q(y)$ into the forward KOLMOGOROV equation (3.5), we obtain for the likelihood ratios of (3.8) the Backward Equation

$$
\partial \ell(t, y)=\sum_{z \in \mathcal{S}} \widehat{\kappa}(y, z) \ell(t, z)=\sum_{z \in \mathcal{S}} \widehat{\kappa}(y, z)[\ell(t, z)-\ell(t, y)]=:(\widehat{\mathcal{K}} \ell)(t, y),
$$

or equivalently $\partial \ell(t)=\widehat{\mathcal{K}} \ell(t)$ in matrix form, with the new transition rates

$$
\widehat{\mathcal{K}}:=(\widehat{\kappa}(y, z))_{(y, z) \in \mathcal{S}^{2}}, \quad \widehat{\kappa}(y, z):=\frac{q(z)}{q(y)} \kappa(z, y) .
$$

The entries of this matrix $\widehat{\mathcal{K}}$ are non-negative off the diagonal, and add up to zero $\sum_{z \in \mathcal{S}} \widehat{\kappa}(y, z)=0$ across every row $y \in \mathcal{S}$, on account of (2.2), (2.9).

We shall think of $(\ell(t))_{0 \leq t<\infty}$ as a curve, now in the space $\mathcal{L}=\mathcal{L}_{+}(\mathcal{S})$ of vectors $\Lambda=(\lambda(x))_{x \in \mathcal{S}}$ with strictly positive elements and $\sum_{x \in \mathcal{S}} q(x) \lambda(x)=1$. These are viewed as likelihood ratios with respect to the invariant distribution and evolving in time via (3.9).

Presently, we shall identify $\widehat{\mathcal{K}}$ of (3.10) with the infinitesimal generator of a suitable continuous-time MARKOV Chain, run backwards in time. A special case, however, is worth mentioning already.

Definition 3.1. Detailed Balance: The invariant distribution $Q$ in (2.2) is said to satisfy the detailedbalance conditions, if

$$
q(y) \kappa(y, z)=q(z) \kappa(z, y), \quad \forall(y, z) \in \mathcal{S}^{2} .
$$

This requirement turns out to be equivalent to the identity $q(y) \varrho_{t}(y, z)=q(z) \varrho_{t}(z, y)$ for all $t \in$ $(0, \infty),(y, z) \in \mathcal{S}^{2} ;$ one leg of the equivalence is immediate, courtesy of (2.12). When (3.11) prevails, $\widehat{\mathcal{K}} \equiv \mathcal{K}$ holds in (3.10); and the backward equation (3.9) for the likelihood ratios $\left(\ell_{t}(x)\right)_{x \in \mathcal{S}}$ of (3.8), is then 
exactly the same as the backward equation (3.1) for $\left(\varrho_{t}(x, y)\right)_{x \in \mathcal{S}}$. We stress that, whenever the detailedbalance conditions (3.11) are needed in the sequel, they will be invoked explicitly.

\section{Discrete Gradient and Divergence; DiRICHLET Form, HiLbERT Norms}

It is apt at this point to introduce some necessary notation and functional-analytic notions. For a given function $f: \mathcal{S} \rightarrow \mathbb{R}$ we consider the discrete gradient $\nabla f: \mathcal{S}^{2} \rightarrow \mathbb{R}$ given by

$$
\nabla f(x, y):=f(y)-f(x) .
$$

In a similar spirit, we consider the discrete divergence

$$
(\nabla \cdot F)(x):=\frac{1}{2} \sum_{y \in \mathcal{S}, y \neq x} \kappa(x, y)[F(x, y)-F(y, x)]
$$

of a function $F: \mathcal{S} \times \mathcal{S} \rightarrow \mathbb{R}$, and note the familiar concatenation formula

$$
\mathcal{K} f=\nabla \cdot(\nabla f)
$$

which allows us to think of the operator $\mathcal{K}$ in (2.14) also as a "discrete Laplacian". We introduce also the set $\mathcal{Z}:=\{(x, y) \in \mathcal{S} \times \mathcal{S}: \kappa(x, y)>0\}$ consisting of all edges in the incidence graph associated with the MARKOV chain, and the measure $C$ on $\mathcal{Z}$ defined by the "conductances"

$$
C\{(x, y)\} \equiv c(x, y):=\frac{1}{2} \kappa(x, y) q(x), \quad(x, y) \in \mathcal{Z} .
$$

With these ingredients, we consider the bilinear forms

$$
\langle f, g\rangle_{\mathbb{L}^{2}(\mathcal{S}, Q)}:=\sum_{x \in \mathcal{S}} q(x) f(x) g(x), \quad\langle F, G\rangle_{\mathbb{L}^{2}(\mathcal{Z}, C)}:=\sum_{(x, y) \in \mathcal{Z}} c(x, y) F(x, y) G(x, y)
$$

for real-valued functions defined on $\mathcal{S}$ (lowercase $f, g$ ) and on $\mathcal{S} \times \mathcal{S}$ (uppercase $F, G$ ), respectively. They induce the $\mathbb{L}^{2}$-norms $\|f\|_{\mathbb{L}^{2}(\mathcal{S}, Q)}$ (relative to the probability measure $Q$ ) and $\|F\|_{\mathbb{L}^{2}(\mathcal{Z}, C)}$ (relative to the unnormalized measure $C$ on $\mathcal{Z}$ in (4.4)), given respectively via

$$
\begin{aligned}
\|f\|_{\mathbb{L}^{2}(\mathcal{S}, Q)}^{2} & :=\langle f, f\rangle_{\mathbb{L}^{2}(\mathcal{S}, Q)}=\sum_{x \in \mathcal{S}} q(x) f^{2}(x), \\
\|F\|_{\mathbb{L}^{2}(\mathcal{Z}, C)}^{2} & :=\langle F, F\rangle_{\mathbb{L}^{2}(\mathcal{Z}, C)}=\sum_{(x, y) \in \mathcal{Z}} c(x, y) F^{2}(x, y) .
\end{aligned}
$$

Remark 4.1. We note from (3.9)-(3.10) the adjoint relationship

$$
\langle f, \widehat{\mathcal{K}} g\rangle_{\mathbb{L}^{2}(\mathcal{S}, Q)}=\langle\mathcal{K} f, g\rangle_{\mathbb{L}^{2}(\mathcal{S}, Q)} .
$$

Thus (3.11) holds if, and only if, the operator $\mathcal{K}$ in (2.14) is self-adjoint on $\mathbb{L}^{2}(\mathcal{S}, Q)$.

Finally, we introduce the bilinear DIRICHLET form associated with the MARKOV Chain:

$$
\mathcal{E}(f, g):=-\langle f, \mathcal{K} g\rangle_{\mathbb{L}^{2}(\mathcal{S}, Q)}=-\sum_{y \in \mathcal{S}} q(y) f(y)(\mathcal{K} g)(y)=-\sum_{x \in \mathcal{S}} \sum_{y \in \mathcal{S}} q(y) \kappa(y, x) f(y) g(x) .
$$

This form is not symmetric, in general; but satisfies $\mathcal{E}(f, f) \geq 0$, as follows from Lemma 4.1 below.

Lemma 4.1. The DIRICHLET form (4.8) can be cast equivalently as

$$
\mathcal{E}(f, g)=\frac{1}{2} \sum_{x \in \mathcal{S}} \sum_{y \in \mathcal{S}} \kappa(y, x) q(y)(f(y)-g(x))^{2} .
$$


Proof: We have clearly $\sum_{x \in \mathcal{S}} \sum_{y \in \mathcal{S}} \kappa(y, x) q(y) f^{2}(y)=0$ on account of $\sum_{x \in \mathcal{S}} \kappa(y, x)=0$ for every $y \in \mathcal{S}$; as well as

$$
\sum_{x \in \mathcal{S}} \sum_{y \in \mathcal{S}} \kappa(y, x) q(y) g^{2}(x)=\sum_{x \in \mathcal{S}} \sum_{y \in \mathcal{S}} \widehat{\kappa}(x, y) q(x) g^{2}(x)=0
$$

from the adjoint rates of (3.10) and their property $\sum_{y \in \mathcal{S}} \widehat{\kappa}(x, y)=0, \forall x \in \mathcal{S}$. It follows from (4.8) that

$$
\sum_{x \in \mathcal{S}} \sum_{y \in \mathcal{S}} \kappa(y, x) q(y)(f(y)-g(x))^{2}=-2 \sum_{x \in \mathcal{S}} \sum_{y \in \mathcal{S}} \kappa(y, x) q(y) f(y) g(x)=2 \mathcal{E}(f, g) .
$$

\subsection{Consequences of Detailed Balance}

The detailed-balance conditions (3.11) can be thought of as positing that "the conductances of (4.4) do not depend on the direction of the current's flow". Under these conditions, we have for functions $f: \mathcal{S} \rightarrow \mathbb{R}$ and $F: \mathcal{S} \times \mathcal{S} \rightarrow \mathbb{R}$ the discrete integration-by-parts formula

$$
\langle\nabla f, F\rangle_{\mathbb{L}^{2}(\mathcal{Z}, C)}=-\langle f, \nabla \cdot F\rangle_{\mathbb{L}^{2}(\mathcal{S}, Q)},
$$

in addition to the concatenation property (4.3). As a result, the bilinear DIRICHLET form of (4.8), (4.9) is now symmetric, and induces the HILBERT $\mathbb{H}^{1}$-inner product and norm

$$
\begin{gathered}
\langle f, g\rangle_{\mathbb{H}^{1}(\mathcal{S}, Q)}:=\mathcal{E}(f, g)=\langle\nabla f, \nabla g\rangle_{\mathbb{L}^{2}(\mathcal{Z}, C)}, \\
\|f\|_{\mathbb{H}^{1}(\mathcal{S}, Q)}^{2}:=\mathcal{E}(f, f)=-\langle f, \mathcal{K} f\rangle_{\mathbb{L}^{2}(\mathcal{S}, Q)}=\sum_{(x, y) \in \mathcal{Z}} c(x, y)(f(y)-f(x))^{2}=\|\nabla f\|_{\mathbb{L}^{2}(\mathcal{Z}, C)}^{2},
\end{gathered}
$$

respectively. We introduce also the dual of this norm, the HILBERT $\mathbb{H}^{-1}-$ norm

$$
\|f\|_{\mathbb{H}^{-1}(\mathcal{S}, Q)}:=\left\|\nabla\left(\mathcal{K}^{-1} f\right)\right\|_{\mathbb{L}^{2}(\mathcal{Z}, C)}, \quad \text { if } f \in \operatorname{Range}(\mathcal{K}) ; \quad\|f\|_{\mathbb{H}^{-1}(\mathcal{S}, Q)}:=+\infty, \quad \text { otherwise; }
$$

and note the variational characterizations

$$
\begin{gathered}
\|f\|_{\mathbb{H}^{-1}(\mathcal{S}, Q)}=\sup _{g: \mathcal{S} \rightarrow \mathbb{R}} \frac{\langle f, g\rangle_{\mathbb{L}^{2}(\mathcal{S}, Q)}}{\|g\|_{\mathbb{H}^{1}(\mathcal{S}, Q)}}, \\
\|f\|_{\mathbb{H}^{-1}(\mathcal{S}, Q)}=\inf _{F: \mathcal{Z} \rightarrow \mathbb{R}}\left\{\|F\|_{\mathbb{L}^{2}(\mathcal{Z}, C)}: f=\nabla \cdot F\right\}=\inf _{g: \mathcal{S} \rightarrow \mathbb{R}}\left\{\|\nabla g\|_{\mathbb{L}^{2}(\mathcal{Z}, C)}: f=\mathcal{K} g\right\} .
\end{gathered}
$$

Basic HILBERT space theory shows that these two infima are attained.

Lemma 4.2. Under the conditions of (3.11), the expression (4.9) for the DIRICHLET form becomes

$$
\mathcal{E}(f, g)=\frac{1}{2} \sum_{x \in \mathcal{S}} \sum_{y \in \mathcal{S}} \kappa(y, x) q(y)[f(y)-f(x)][g(y)-g(x)]=\langle\nabla f, \nabla g\rangle_{\mathbb{L}^{2}(\mathcal{Z}, C)}=\langle f, g\rangle_{\mathbb{H}^{1}(\mathcal{S}, Q)} .
$$

Proof: Let us write the double summation in the above display as

$$
\begin{gathered}
\sum_{x \in \mathcal{S}} \sum_{y \in \mathcal{S}} \kappa(y, x) q(y)[f(y) g(y)-f(y) g(x)-f(x) g(y)+f(x) g(x)]= \\
=-\sum_{x \in \mathcal{S}} \sum_{y \in \mathcal{S}} \kappa(y, x) q(y)[f(y) g(x)+f(x) g(y)]=-2 \sum_{x \in \mathcal{S}} \sum_{y \in \mathcal{S}} \kappa(y, x) q(y) f(y) g(x)=-2 \mathcal{E}(f, g) .
\end{gathered}
$$


Here, the first equality uses (3.10), as well as the properties $\sum_{x \in \mathcal{S}} \kappa(y, x)=0$ for every $y \in \mathcal{S}$, and $\sum_{y \in \mathcal{S}} \widehat{\kappa}(x, y)=0$ for every $x \in \mathcal{S}$; whereas, the second equality uses the conditions (3.11), and the third equality is just (4.8).

This proves the first equality in (4.16). The second and third are just restatements of (4.11).

Remark 4.2. Additional Consequences: It follows from (4.10)-(4.12) that, under the detailed-balance conditions (3.11), the mapping

$$
\nabla: \mathbb{H}^{1}(\mathcal{S}, Q) \rightarrow \mathbb{L}^{2}(\mathcal{Z}, C)
$$

is an isometric embedding. Whereas, the discrete divergence mapping $\nabla \cdot$ in (4.2) is, up to a minus sign, the adjoint of the mapping $\nabla: \mathbb{L}^{2}(\mathcal{S}, Q) \rightarrow \mathbb{L}^{2}(\mathcal{Z}, C)$.

Remark 4.3. A Counterexample. In the absence of detailed balance, the DIRICHLET form $\mathcal{E}(f, g)$ is not an inner product; indeed, Remark 4.1 shows that there exist functions $f: \mathcal{S} \rightarrow \mathbb{R}, g: \mathcal{S} \rightarrow \mathbb{R}$ with $\mathcal{E}(f, g)=$ $-\langle f, \mathcal{K} g\rangle_{\mathbb{L}^{2}(\mathcal{S}, Q)} \neq-\langle g, \mathcal{K} f\rangle_{\mathbb{L}^{2}(\mathcal{S}, Q)}=\mathcal{E}(g, f)$. An explicit example of this situation is provided by the matrix

$$
\mathcal{K}=\left(\begin{array}{ccc}
-1 & 1 & 0 \\
0 & -1 & 1 \\
1 & 0 & -1
\end{array}\right)
$$

whose invariant distribution $Q=(1 / 3,1 / 3,1 / 3)$ is uniform on the state space $\mathcal{S}=\{1,2,3\}$ and for which detailed balance fails. Whereas, with $f=\mathfrak{e}_{1}=(1,0,0)$ and $g=\mathfrak{e}_{2}=(0,1,0)$ the first and second unit row vectors, respectively, and noting $3 \mathcal{E}(\varphi, \gamma)=\varphi \mathcal{K}^{\prime} \gamma^{\prime}$ from (4.8), we observe

$$
3 \mathcal{E}(f, g)=(1,0,0)\left(\begin{array}{c}
1 \\
-1 \\
0
\end{array}\right)=-1, \quad 3 \mathcal{E}(g, f)=(0,1,0)\left(\begin{array}{c}
-1 \\
0 \\
1
\end{array}\right)=0 .
$$

Nevertheless, $\|f\|_{\mathbb{H}^{1}(\mathcal{S}, Q)}=\sqrt{\mathcal{E}(f, f)}$ is always a HILBERT norm, with associated inner product given by the DIRICHLET form $\mathcal{E}_{\text {sym }}(f, g)$ of the reversible MARKOV Chain, with symmetrized rates $\kappa_{\text {sym }}(x, y):=$ $(\kappa(x, y)+\widehat{\kappa}(x, y)) / 2$ in the manner of (4.8), (3.10); namely, $\mathcal{E}_{\text {sym }}(f, f) \equiv \mathcal{E}(f, f)$ and

$$
\langle f, g\rangle_{\mathbb{H}^{1}(\mathcal{S}, Q)}=-\frac{1}{2} \sum_{x \in \mathcal{S}} \sum_{y \in \mathcal{S}}[q(y) \kappa(y, x)+q(x) \kappa(x, y)] f(x) g(y)=-\sum_{x \in \mathcal{S}} \sum_{y \in \mathcal{S}} q(y) \kappa_{\mathrm{sym}}(y, x) f(y) g(x) .
$$

\section{Time Reversal and Associated Martingales}

It is well known that the MARKOV property is invariant under reversal of time (interchanging the roles of "past" and "future", keeping the "present" as is). This means, in particular, that the time-reversed process

$$
\widehat{X}(s):=X(T-s), \quad 0 \leq s \leq T
$$

is a MARKOV Chain, for any given $T \in(0, \infty)$. But how about the transition probabilities of this timereversed process? These are fairly easy to compute, namely,

$$
\mathbb{P}\left(\widehat{X}\left(s_{2}\right)=z \mid \widehat{\mathcal{G}}\left(s_{1}\right)\right)=\mathbb{P}\left(\widehat{X}\left(s_{2}\right)=z \mid \widehat{X}\left(s_{1}\right)\right)=\rho^{*}\left(s_{1}, \widehat{X}\left(s_{1}\right) ; s_{2}, z\right)
$$

for $0 \leq s_{1} \leq s_{2} \leq T, z \in \mathcal{S}$, where

$$
\rho^{*}\left(s_{1}, y ; s_{2}, z\right):=\frac{p\left(T-s_{2}, z\right)}{p\left(T-s_{1}, y\right)} \varrho_{s_{2}-s_{1}}(z, y) ;
$$

but need not be time-homogeneous in general. 
However: Let us compute these same transition probabilities when the Chain starts at its invariant distribution $Q$. We introduce at this point another probability measure $\mathbb{Q}$ on the underlying measurable space $(\Omega, \mathcal{F})$, under which the MARKOV Chain $\mathcal{X}$ has exactly the same dynamics as before, but its initial distribution is the invariant probability vector $Q=(q(y))_{y \in \mathcal{S}}$ in (2.2). Then, in lieu of (2.6), the finitedimensional distributions of the Chain are

$$
\begin{aligned}
\mathbb{Q}\left(X(0)=x, X\left(\theta_{1}\right)\right. & \left.=y_{1}, \cdots, X\left(\theta_{n}\right)=y_{n}, X(t)=z\right)= \\
= & q(x) \varrho_{\theta_{1}}\left(x, y_{1}\right) \varrho_{\theta_{2}-\theta_{1}}\left(y_{1}, y_{2}\right) \cdots \varrho_{\theta_{n}-\theta_{n-1}}\left(y_{n-1}, y_{n}\right) \cdot \varrho_{t-\theta}(y, z) .
\end{aligned}
$$

On each $\sigma$-algebra $\mathcal{F}^{X}(t), 0 \leq t<\infty$, the two probability measures $\mathbb{P}$ and $\mathbb{Q}$ are equivalent; in fact, on the smaller $\sigma$-algebra $\sigma(X(t))$, we single out in the notation of (3.8) the so-called likelihood process

$$
L(t):=\left.\frac{\mathrm{d} \mathbb{P}}{\mathrm{d} \mathbb{Q}}\right|_{\sigma(X(t))}=\ell(t, X(t)), \quad 0 \leq t<\infty .
$$

Under this dispensation, the transition probabilities are

$$
\mathbb{Q}\left(\widehat{X}\left(s_{2}\right)=z \mid \widehat{\mathcal{G}}\left(s_{1}\right)\right)=\mathbb{Q}\left(\widehat{X}\left(s_{2}\right)=z \mid \widehat{X}\left(s_{1}\right)\right)=\widehat{\varrho}_{s_{2}-s_{1}}\left(\widehat{X}\left(s_{1}\right), z\right),
$$

i.e., time-homogeneous, with

$$
\widehat{\varrho}_{h}(y, z):=\frac{q(z)}{q(y)} \varrho_{h}(z, y) .
$$

Invoking (5.6) and (2.12), we see that the $\mathbb{Q}$-infinitesimal-generator of this time-reversed MARKOV Chain $\widehat{X}(s)=X(T-s), 0 \leq s \leq T$ in (5.1), is given precisely by $\widehat{\mathcal{K}}=(\widehat{\kappa}(y, z))_{(y, z) \in \mathcal{S}^{2}}$ as in (3.10). (We note parenthetically that, when the detailed-balance conditions (3.11) hold, the initial distributions and transition probabilities of the continuous-time MARKOV Chain $X(t), 0 \leq t \leq T$, and of its time-reversal (5.1), are exactly the same under the probability measure $\mathbb{Q}$.)

Remark 5.1. The standing assumption $P(0) \in \mathcal{M}$, i.e., that all entries of the initial distribution are strictly positive, is made for economy of exposition. For even when the probability vector $P(0)$ belongs to the closure $\overline{\mathcal{M}}$ of $\mathcal{M}$, i.e., some of its entries are allowed to vanish, there is at least one $x \in \mathcal{S}$ with $p(0, x)>0$; then (3.4) and irreducibility imply $p(t, y)>0$ for all $t>0, y \in \mathcal{S}$. Thus, even if the curve $(P(t))_{0 \leq t<\infty}$ starts out on the boundary $\overline{\mathcal{M}} \backslash \mathcal{M}$, it enters $\mathcal{M}$ immediately and stays there for all times $t \in(0, \infty)$.

By complete analogy with Proposition 2.1, we formulate now the following result.

Proposition 5.1. For any given function $g:[0, T] \times \mathcal{S} \rightarrow \mathbb{R}$ whose temporal derivative $s \mapsto \partial g(s, x)$ is continuous for every state $x \in \mathcal{S}$, the process below is a $(\widehat{\mathbb{G}}, \mathbb{Q})$-local martingale:

$$
\widehat{M}^{g}(s):=g(s, \widehat{X}(s))-\int_{0}^{s}(\partial g+\widehat{\mathcal{K}} g)(u, \widehat{X}(u)) \mathrm{d} u, \quad 0 \leq s \leq T .
$$

The following important result is due to PAVON (1989), FONTBONA \& JOURDAIN (2016) in the context of diffusions. Its proof (cf. Theorem 4.2 in KARATZAS, SCHACHERMAYER \& TSCHIDERER (2019)) uses only the MARKOV property and the definition of conditional expectation, and carries over verbatim to our present context. An alternative argument, specific to the MARKOV Chain context, uses Proposition 5.1 and is given right below.

Proposition 5.2. Time-Reversed Likelihood Process as Martingale: Fix $T \in(0, \infty)$ and consider the time-reversed Chain (5.1), as well as the filtration $\widehat{\mathbb{G}}=\{\widehat{\mathcal{G}}(s)\}_{0 \leq s \leq T}$ this process generates via $\widehat{\mathcal{G}}(s):=$ $\sigma(\widehat{X}(u), 0 \leq u \leq s)$. Then, the time-reversed likelihood process

$$
L(T-s)=\ell(T-s, \widehat{X}(s)), \quad 0 \leq s \leq T \quad \text { is } a(\widehat{\mathbb{G}}, \mathbb{Q})-\text { martingale. }
$$


Proof: We consider in (5.7) the function $g(s, x)=\ell(T-s, x), 0 \leq s \leq T, x \in \mathcal{S}$ and note that $\partial g(s, x)=-\partial \ell(T-s, x)=-(\widehat{\mathcal{K}} \ell)(T-s, x)$ holds on account of (3.9).

It follows from (5.7), whose integrand now vanishes, that the time-reversed likelihood ratio process $\ell(T-s, \widehat{X}(s)), 0 \leq s \leq T$ is a $\mathbb{Q}$-local-martingale of the time-reversed filtration $\widehat{\mathbb{G}}$. But this process is positive, thus also a $\mathbb{Q}$-supermartingale, and its expectation

$$
\mathbb{E}^{\mathbb{Q}}[\ell(T-s, X(T-s))]=\sum_{y \in \mathcal{S}} q(y) \frac{p(T-s, y)}{q(y)}=1, \quad 0 \leq s \leq T
$$

is constant. Therefore $\ell(T-s, \widehat{X}(s)), 0 \leq s \leq T$ is a true $\mathbb{Q}$-martingale, exactly as stated in (5.8).

\section{The Variance Process}

For a probability vector $P=(p(y))_{y \in \mathcal{S}} \in \mathcal{M}$ with positive entries, we introduce its likelihood vector $\ell=(\ell(y))_{y \in \mathcal{S}} \in \mathcal{L}$ with $\ell(y)=p(y) / q(y)$ as in (3.8), relative to the invariant distribution $Q$ of the Chain. We define then in the manner of (4.6) the Variance of $P$ relative to $Q$, also known as $\chi^{2}$-divergence, as

$$
V(P \mid Q) \equiv \operatorname{Var}^{\mathbb{Q}}(\ell):=\sum_{y \in \mathcal{S}} q(y) \ell^{2}(y)-1=\|\ell\|_{\mathbb{L}^{2}(\mathcal{S}, Q)}^{2}-1 .
$$

Let us recall now from (3.4) the curve $(P(t))_{0 \leq t<\infty} \subset \mathcal{M}$ of time-marginal distributions for our continuoustime MARKov Chain, and the corresponding curve of likelihoods $\ell_{t}=(\ell(t, y))_{y \in \mathcal{S}}, 0 \leq t<\infty$ in the space $\mathcal{L}$, with $\ell(t, y)=p(t, y) / q(y)$. We will show in Proposition 6.2 that the variance just defined in $(6.1)$ plays the role of LYAPUNOV function for the convergence to equilibrium along this curve.

To see this, we summon the likelihood process $L(t)=\ell(t, X(t)), 0 \leq t<\infty$ from (5.4) and consider its square $L^{2}(t), 0 \leq t<\infty$, the so-called Variance Process, under time-reversal.

Proposition 6.1. For any given $T \in(0, \infty)$, we have the DOOB-MEYER decomposition

$$
\ell^{2}(T-s, \widehat{X}(s))=\widehat{M}(s)+\left.\int_{0}^{s} \sum_{y \neq x}\left(\widehat{\kappa}(x, y)(\ell(t, y)-\ell(t, x))^{2}\right)\right|_{\substack{t=T-u \\ x=\widehat{X}(u)}} \mathrm{d} u, \quad 0 \leq s \leq T
$$

of the time-reversed variance process $\ell^{2}(T-s, \widehat{X}(s)), 0 \leq s \leq T$, where $\widehat{M}$ is a $(\widehat{\mathbb{G}}, \mathbb{Q})$-martingale.

Proof: The first claim follows from Proposition 5.2 and the JENSEN inequality. For the second claim we deploy Proposition 5.1 with $g(s, x):=\ell^{2}(T-s, x), 0 \leq s \leq T, x \in \mathcal{S}$, to conclude via the calculation

$$
(\partial g+\widehat{\mathcal{K}} g)(T-s, x)=\sum_{y \in \mathcal{S}} \widehat{\kappa}(x, y)(\ell(T-s, y)-\ell(T-s, x))^{2}
$$

that $\widehat{M}$ is a local $(\widehat{\mathbb{G}}, \mathbb{Q})$-martingale. The uniform continuity of $[0, T] \ni t \mapsto p_{t}(x, y) \in[0,1]$ and the finiteness of the state-space imply that this process is actually bounded, thus a true $\mathbb{Q}$-martingale.

Let us now justify the claim (6.3). From the Backwards Equation (3.9), we have

$$
\begin{gathered}
\partial g(T-s, x)=-2 \ell(T-s, x) \partial \ell(T-s, x)=-2 \ell(T-s, x) \sum_{y \in \mathcal{S}} \widehat{\kappa}(x, y) \ell(T-s, y), \\
(\widehat{\mathcal{K}} g)(T-s, x)=\sum_{y \in \mathcal{S}} \widehat{\kappa}(x, y) \ell^{2}(T-s, y)=\sum_{y \in \mathcal{S}} \widehat{\kappa}(x, y)\left[\ell^{2}(T-s, y)+\ell^{2}(T-s, x)\right]
\end{gathered}
$$


on account of the property $\sum_{y \in \mathcal{S}} \widehat{\kappa}(x, y)=0$ for every $x \in \mathcal{S}$; now (6.3) follows readily.

Proposition 6.1 deals with the trajectorial behavior of the variance process; and for this, it is crucial to let time run backwards. Now, we want to adopt also an "aggregate" point of view, and take $\mathbb{Q}$-expectations in (6.2). When doing this, it does not matter any more whether time runs forwards or backwards, so we state the following result "forwards in time". Recalling (3.10), we obtain thus the dissipation of the variance.

Proposition 6.2. Along the curve $(P(t))_{0 \leq t<\infty}$ of time-marginal distributions in (3.4), the variance

$$
V(P(t) \mid Q)=\operatorname{Var}^{\mathbb{Q}}\left(\ell_{t}\right)=\sum_{y \in \mathcal{S}} q(y) \ell^{2}(t, y)-1=\left\|\ell_{t}\right\|_{\mathbb{L}^{2}(\mathcal{S}, Q)}^{2}-1, \quad 0 \leq t<\infty
$$

is decreasing with $\lim _{t \rightarrow \infty} \downarrow V(P(t) \mid Q)=0$, and the rate of its decrease is given by

$$
\partial\left\|\boldsymbol{\ell}_{t}\right\|_{\mathbb{L}^{2}(\mathcal{S}, Q)}^{2}=\partial V(P(t) \mid Q)=-2 \mathcal{E}\left(\boldsymbol{\ell}_{t}, \boldsymbol{\ell}_{t}\right)
$$

(thus by $-2\left\|\ell_{t}\right\|_{\mathbb{H}^{1}(\mathcal{S}, Q)}^{2}$ under the detailed-balance conditions (3.11)). More precisely,

$$
\begin{aligned}
V(P(T) \mid Q) & =V(P(0) \mid Q)-\int_{0}^{T} \sum_{(x, y) \in \mathcal{Z}} q(y) \kappa(y, x)(\ell(t, y)-\ell(t, x))^{2} \mathrm{~d} t \\
& =\int_{T}^{\infty} \sum_{(x, y) \in \mathcal{Z}} q(y) \kappa(y, x)(\ell(t, y)-\ell(t, x))^{2} \mathrm{~d} t .
\end{aligned}
$$

The decomposition (6.2) is a trajectorial version of this variance dissipation, at the level of the individual particle viewed under the probability measure $\mathbb{Q}$ and under time-reversal. As a consequence of (6.2) and of the BAYES rule, we deduce from (6.2) the DOOB-MEYER decomposition

$$
\ell(T-s, \widehat{X}(s))=\widehat{N}(s)+\left.\int_{0}^{s} \sum_{y \neq x}\left(\frac{\widehat{\kappa}(x, y)}{\ell(t, x)}(\ell(t, y)-\ell(t, x))^{2}\right)\right|_{\substack{t=T-u \\ x=\widehat{X}(u)}} \mathrm{d} u, \quad 0 \leq s \leq T
$$

of the time-reversed likelihood process, where $\widehat{N}$ is a $(\widehat{\mathbb{G}}, \mathbb{P})$-martingale.

\subsection{Steepest Descent of the Variance, under Detailed Balance}

We state now and establish the following result, Theorem 6.3. As pointed out in JORDAN, KINDERLEHRER \& OTTO (1998), results of this type go as far back as the paper by COURANT, FRIEDRICHS \& LEWY (1928) in the Brownian motion context. We deploy the notation of (3.8) for the likelihood ratios relative to the invariant distribution, as well as the following notion.

Definition 6.1. We say that a smooth curve of probability measures $(P(t))_{t_{0} \leq t<\infty} \subset \mathcal{M}=\mathcal{P}_{+}(\mathcal{S})$ is of steepest descent locally at $t=t_{0}$, for a given smooth functional $F: \mathcal{M} \rightarrow \mathbb{R}$ and relative to a given metric $\varrho$ on $\mathcal{M}$, if it minimizes, among all curves $(\widetilde{P}(t))_{t_{0} \leq t<\infty} \subset \mathcal{M}$ satisfying $\widetilde{P}\left(t_{0}\right)=P\left(t_{0}\right)$, the infinitesimal rate of change of $F$ as measured on $\mathcal{M}$ in terms of $\varrho$, namely,

$$
\lim _{h \downarrow 0} \frac{F\left(\widetilde{P}\left(t_{0}+h\right)\right)-F\left(P\left(t_{0}\right)\right)}{\varrho\left(\widetilde{P}\left(t_{0}+h\right), P\left(t_{0}\right)\right)} .
$$


Theorem 6.3. Steepest Descent for the Variance: Under the conditions (3.11) of detailed balance, the curve $(P(t))_{0 \leq t<\infty}$ of time-marginal distributions in (3.4) has the property of steepest decent for the variance of (6.5) with respect to the metric distance bequeathed by the norm of (4.13), i.e.,

$$
\varrho\left(P_{1}, P_{2}\right):=\left\|\ell_{1}-\ell_{2}\right\|_{\mathbb{H}^{-1}(\mathcal{S}, Q)} \quad \text { for } P_{1}=\ell_{1} Q \text { and } P_{2}=\ell_{2} Q .
$$

The proof of this result needs Proposition 6.5 below. We pave the way towards it by formulating first a variational version of Propositions 6.1, 6.2. For this purpose, we fix an arbitrary time-point $t_{0} \in(0, \infty)$ and let $\psi(\cdot)=(\psi(t))_{t_{0} \leq t<t_{0}+\varepsilon}$ be a continuous curve of real-valued functions on the state-space $\mathcal{S}$.

With these ingredients, we define a new curve $\ell^{\psi}(\cdot)=\left(\ell^{\psi}(t)\right)_{t_{0} \leq t<t_{0}+\varepsilon}$ of such functions, for a suitable $\varepsilon>0$, by specifying in the space $\mathcal{L}=\mathcal{L}_{+}(\mathcal{S})$ of subsection 3.2 the initial condition $\ell^{\psi}\left(t_{0}\right)=\ell\left(t_{0}\right) \in \mathcal{L}$ and the dynamics $\partial \ell^{\psi}(t)=(\widehat{\mathcal{K}} \psi)(t)$ for $t \in\left[t_{0}, t_{0}+\varepsilon\right)$; in the manner of (3.9) and a bit more explicitly,

$$
\partial \ell^{\psi}(t, x)=\sum_{y \in \mathcal{S}} \widehat{\kappa}(x, y) \psi(t, y), \quad x \in \mathcal{S} .
$$

The curve $\ell^{\psi}(\cdot)=\left(\ell^{\psi}(t)\right)_{t_{0} \leq t<t_{0}+\varepsilon}$, the "output" of the system (6.11) corresponding to the "input" $\psi(\cdot)$, is only defined on an interval $\left[t_{0}, t_{0}+\varepsilon\right)$ and lives in the space $\mathcal{L}$, since

$$
\partial \sum_{x \in \mathcal{S}} q(x) \ell^{\psi}(t, x)=\sum_{x \in \mathcal{S}} q(x) \sum_{y \in \mathcal{S}} \widehat{\kappa}(x, y) \psi(t, y)=\sum_{y \in \mathcal{S}} \sum_{x \in \mathcal{S}} q(y) \kappa(y, x) \psi(t, y)=0
$$

implies $\sum_{x \in \mathcal{S}} q(x) \ell^{\psi}(t, x)=\sum_{x \in \mathcal{S}} q(x) \boldsymbol{\ell}\left(t_{0}, x\right)=1$ for all $t \in\left[t_{0}+\varepsilon\right)$. Thus, the recipe

$$
p^{\psi}(t, x):=q(x) \ell^{\psi}(t, x), \quad(t, x) \in\left[t_{0}, t_{0}+\varepsilon\right) \times \mathcal{S}
$$

procures a curve $\left(P^{\psi}(t)\right)_{0 \leq t<t_{0}+\varepsilon}$, on the manifold $\mathcal{M}=\mathcal{P}_{+}(\mathcal{S})$ in subsection 3.1 consisting of vectors $P=(p(x))_{x \in \mathcal{S}}$ with strictly positive elements and total mass $\sum_{x \in \mathcal{S}} p(x)=1$.

Conversely: By irreducibility, the "input curve" $\psi(\cdot)$ is determined by the "output curve" $\ell^{\psi}(\cdot)$ up to an additive time-dependent constant. In particular, every smooth curve $\ell^{*}(\cdot)=\left(\ell^{*}(t)\right)_{t_{0} \leq t<t_{0}+\varepsilon}$ in $\mathcal{L}$ with $\ell^{*}\left(t_{0}\right)=\ell\left(t_{0}\right)$ is representable as $\ell^{\psi}(\cdot)$ for a suitable continuous $\psi(\cdot)$ as above. For instance, $\ell(\cdot) \in \mathcal{L}$ of (3.8) is the "output" that corresponds in this manner to the "input" $\psi(\cdot) \equiv \boldsymbol{\ell}(\cdot)$ in (6.11), via (3.9).

We have the following generalization of Proposition 6.2, to which it reduces when $\psi(\cdot) \equiv \ell(\cdot)$.

Proposition 6.4. In the above context, we have for $t \in\left[t_{0}+\varepsilon\right)$ the properties

$$
\partial V\left(P^{\psi}(t) \mid Q\right)=\partial \mathbb{E}^{\mathbb{Q}}\left[\left(\ell^{\psi}\right)^{2}(t, X(t))\right]=2\left\langle\psi_{t}, \mathcal{K} \ell_{t}^{\psi}\right\rangle_{\mathbb{L}^{2}(\mathcal{S}, Q)}=-2 \mathcal{E}\left(\psi_{t}, \ell_{t}^{\psi}\right) .
$$

Whereas, under the detailed balance conditions (3.11), this expression becomes

$$
\partial V\left(P^{\psi}(t) \mid Q\right)=-2 \mathcal{E}\left(\ell_{t}^{\psi}, \psi_{t}\right)=-2\left\langle\nabla \ell_{t}^{\psi}, \nabla \psi_{t}\right\rangle_{\mathbb{L}^{2}(\mathcal{Z}, C)}=-2\left\langle\ell_{t}^{\psi}, \psi_{t}\right\rangle_{\mathbb{H}^{1}(\mathcal{S}, Q)} .
$$

Proof: A reasoning similar to that in Propositions 6.1 and 6.2, and carried out once again in the backwards direction of time, can be deployed by applying Proposition 5.1 to $g(s, x):=\left(\ell^{\psi}\right)^{2}(T-s, x), \quad 0 \leq s \leq T$, $x \in \mathcal{S}$ for arbitrary but fixed $T \in\left(0, t_{0}+\varepsilon\right)$. But here is a simpler argument:

$$
\partial V\left(P^{\psi}(t) \mid Q\right)=\partial\left\|\ell_{t}^{\psi}\right\|_{\mathbb{L}^{2}(\mathcal{S}, Q)}^{2}=2\left\langle\ell_{t}^{\psi}, \widehat{\mathcal{K}} \psi_{t}\right\rangle_{\mathbb{L}^{2}(\mathcal{S}, Q)}=2\left\langle\psi_{t}, \mathcal{K} \ell_{t}^{\psi}\right\rangle_{\mathbb{L}^{2}(\mathcal{S}, Q)}=-2 \mathcal{E}\left(\psi_{t}, \ell_{t}^{\psi}\right),
$$

on account of (4.7), (4.8) and (3.10). This reasoning proves Proposition 6.2 as well.

We compute now the "infinitesimal cost of moving the curve" $\left(\ell^{\psi}(t)\right)_{t_{0} \leq t<t_{0}+\varepsilon}$. 
Proposition 6.5. Under the conditions (3.11) of detailed balance, we have

$$
\lim _{h \downarrow 0} \frac{1}{h}\left\|\boldsymbol{\ell}_{t+h}-\boldsymbol{\ell}_{t}\right\|_{\mathbb{H}^{-1}(\mathcal{S}, Q)}=\left\|\mathcal{K} \boldsymbol{\ell}_{t}\right\|_{\mathbb{H}^{-1}(\mathcal{S}, Q)}=\left\|\boldsymbol{\ell}_{t}\right\|_{\mathbb{H}^{1}(\mathcal{S}, Q)}
$$

for every $t \in\left[t_{0}, t_{0}+\varepsilon\right)$; and a bit more generally, in the notation just developed,

$$
\lim _{h \downarrow 0} \frac{1}{h}\left\|\ell_{t+h}^{\psi}-\ell_{t}^{\psi}\right\|_{\mathbb{H}^{-1}(\mathcal{S}, Q)}=\left\|\mathcal{K} \psi_{t}\right\|_{\mathbb{H}^{-1}(\mathcal{S}, Q)}=\left\|\psi_{t}\right\|_{\mathbb{H}^{1}(\mathcal{S}, Q)} .
$$

Proof: From (6.11), (3.11) it follows that for every $x \in \mathcal{S}$ we have

$$
\lim _{h \downarrow 0} \frac{1}{h}\left[\ell_{t+h}^{\psi}(x)-\ell_{t}^{\psi}(x)\right]=\left(\mathcal{K} \psi_{t}\right)(x),
$$

so the first equality in (6.14) is evident. For the second equality in (6.14) it suffices to recall (4.13)-(4.15), observe that $\nabla \psi_{t}$ is the unique element $F \in \mathbb{L}^{2}(\mathcal{Z}, C)$ with the property $\nabla \cdot F=\mathcal{K} \psi_{t}$, and note from Remark 4.2 the isometry $\|F\|_{\mathbb{L}^{2}(\mathcal{Z}, C)}=\left\|\psi_{t}\right\|_{\mathbb{H}^{1}(\mathcal{S}, Q)}$ from the space $\mathbb{L}^{2}(\mathcal{Z}, C)$ to $\mathbb{H}^{1}(\mathcal{S}, Q)$.

Now, (6.13) is just a special case of (6.14) with $\psi(\cdot) \equiv \ell(\cdot)$, as discussed above.

\subsection{The Proof of Theorem 6.3}

We are ready to tackle the proof of Theorem 6.3. Along any smooth curve of the form $\left(P^{\psi}(t)\right)_{t_{0} \leq t<t_{0}+\varepsilon}$ created as in (6.11), (6.12) on the manifold of probability vectors $\mathcal{M}=\mathcal{P}_{+}(\mathcal{S})$ and with $\ell^{\psi}\left(t_{0}\right)=\ell\left(t_{0}\right) \in$ $\mathcal{L}$, we have from Propositions 6.4, 6.5 the respective rates for the variance and the metric distance, under detailed balance:

$$
\begin{gathered}
\lim _{h \downarrow 0} \frac{V\left(P^{\psi}\left(t_{0}+h\right) \mid Q\right)-V\left(P\left(t_{0}\right) \mid Q\right)}{h}=-2\left\langle\ell_{t_{0}}, \psi_{t_{0}}\right\rangle_{\mathbb{H}^{1}(\mathcal{S}, Q)}, \\
\lim _{h \downarrow 0} \frac{\varrho\left(P^{\psi}\left(t_{0}+h\right), P\left(t_{0}\right)\right)}{h}=\left\|\psi_{t_{0}}\right\|_{\mathbb{H}^{1}(\mathcal{S}, Q)} .
\end{gathered}
$$

Thus, the rate of change for the variance along the perturbed curve $\left(P^{\psi}(t)\right)_{t_{0} \leq t<t_{0}+\varepsilon}$, when measured on the manifold $\mathcal{M}$ by the metric distance in (6.10), is

$$
\lim _{h \downarrow 0} \frac{V\left(P^{\psi}\left(t_{0}+h\right) \mid Q\right)-V\left(P\left(t_{0}\right) \mid Q\right)}{\varrho\left(P^{\psi}\left(t_{0}+h\right), P\left(t_{0}\right)\right)}=-2\left\langle\ell_{t_{0}}, \frac{\psi_{t_{0}}}{\left\|\psi_{t_{0}}\right\|_{\mathbb{H}^{1}(\mathcal{S}, Q)}}\right\rangle_{\mathbb{H}^{1}(\mathcal{S}, Q)} .
$$

On the other hand, along the original curve $(P(t))_{0 \leq t<\infty}$ of time-marginal distributions for the Chain (that is, with $\psi(\cdot) \equiv \ell(\cdot)$ modulo an affine transformation, as noted above), the rate of variance dissipation measured in terms of the metric distance traveled on the manifold $\mathcal{M}$ is

$$
\lim _{h \downarrow 0} \frac{V\left(P\left(t_{0}+h\right) \mid Q\right)-V\left(P\left(t_{0}\right) \mid Q\right)}{\varrho\left(P\left(t_{0}+h\right), P\left(t_{0}\right)\right)}=-2\left\|\ell_{t_{0}}\right\|_{\mathbb{H}^{1}(\mathcal{S}, Q)}<0 .
$$

A simple comparison of the last two displays, via CAUCHY-SCHWARZ, gives the steepest descent property of the variance with respect to the metric distance in (6.10), i.e.,

$$
\begin{gathered}
\lim _{h \downarrow 0} \frac{V\left(P^{\psi}\left(t_{0}+h\right) \mid Q\right)-V\left(P\left(t_{0}\right) \mid Q\right)}{\varrho\left(P^{\psi}\left(t_{0}+h\right), P\left(t_{0}\right)\right)}-\lim _{h \downarrow 0} \frac{V\left(P\left(t_{0}+h\right) \mid Q\right)-V\left(P\left(t_{0}\right) \mid Q\right)}{\varrho\left(P\left(t_{0}+h\right), P\left(t_{0}\right)\right)} \\
=2\left(\left\|\ell_{t_{0}}\right\|_{\mathbb{H}^{1}(\mathcal{S}, Q)}-\left\langle\boldsymbol{\ell}_{t_{0}}, \frac{\psi_{t_{0}}}{\left\|\psi_{t_{0}}\right\|_{\mathbb{H}^{1}(\mathcal{S}, Q)}}\right\rangle_{\mathbb{H}^{1}(\mathcal{S}, Q)}\right) \geq 0,
\end{gathered}
$$

along the original curve $(P(t))_{0 \leq t<\infty}$ of time-marginals for the MARKOV Chain. Equality holds here if, and only if, $c+\psi_{t_{0}}$ is a positive constant multiple of $\boldsymbol{\ell}_{t_{0}}$ for some $c \in \mathbb{R}$.

We will revisit this theme in Sections 8 and 9. 


\section{The Relative Entropy Process}

For an arbitrary probability vector $P=(p(x))_{x \in \mathcal{S}}$ with strictly positive elements, let us recall the definition of its relative entropy, or KULLBACK-LEIBLER divergence,

$$
H(P \mid Q):=\sum_{x \in \mathcal{S}} p(x) \log \left(\frac{p(x)}{q(x)}\right)
$$

with respect to the invariant distribution $Q=(q(x))_{x \in \mathcal{S}}$ of (2.2). In terms of the likelihood function in (3.8), the relative entropy of the probability vector $P(t)$ in (3.4) with respect to $Q$, is

$$
H(P(t) \mid Q)=\mathbb{E}^{\mathbb{P}}[\log \ell(t, X(t))], \quad 0 \leq t<\infty,
$$

the $\mathbb{P}$-expectation of the log-likelihood at time $t$. We shall see presently that this function

$$
t \longmapsto H(P(t) \mid Q) \text { is non-negative, and satisfies } \lim _{t \rightarrow \infty} \downarrow H(P(t) \mid Q)=0 .
$$

In other words, the relative entropy functional of (7.1) is a LYAPUNOV function for the curve $(P(t))_{0 \leq t<\infty}$ of time-marginal distributions for our continuous-time MARKOV Chain. We shall compute in subsection 7.2 the rate of temporal decrease for the function in (7.3). Of course, all this is in accordance with general thermodynamic principles governing the approach to equilibrium in physical systems (e.g., Chapter 2 in COVER \& THOMAS (1991) in the discrete-time MARKOV Chain context of our Section 2).

Let us note also, that the relative entropy in (7.2) can be cast equivalently as the $\mathbb{Q}$-expectation

$$
H(P(t) \mid Q)=\sum_{y \in \mathcal{S}} q(y) \frac{p(t, y)}{q(y)} \log \left(\frac{p(t, y)}{q(y)}\right)=\mathbb{E}^{\mathbb{Q}}[\ell(t, X(t)) \log \ell(t, X(t))]
$$

of the relative entropy process $\ell(t, X(t)) \cdot \log \ell(t, X(t)), 0 \leq t<\infty$. This allows us to justify the first claim in (7.3), regarding non-negativity. Indeed, the convexity of the function $(0, \infty) \ni \ell \mapsto \Phi(\ell):=$ $\ell \log \ell$ gives, on the strength of the JENSEN inequality,

$$
H(P(t) \mid Q)=\mathbb{E}^{\mathbb{Q}}[\Phi(\ell(t, X(t)))] \geq \Phi\left(\mathbb{E}^{\mathbb{Q}}[\ell(t, X(t))]\right)=f(1)=0 .
$$

Alternatively, this follows from $H(P(t) \mid Q)=\mathbb{E}^{\mathbb{Q}}[\Psi(\ell(t, X(t)))]$, with $\Psi \geq 0$ as in (7.13) below.

Proposition 7.1. In the context of Proposition 5.2, the time-reversed relative entropy process

$$
\ell(T-s, \widehat{X}(s)) \cdot \log \ell(T-s, \widehat{X}(s)), \quad 0 \leq s \leq T \quad \text { is } a(\widehat{\mathbb{G}}, \mathbb{Q})-\text { submartingale; }
$$

the properties in (7.3) hold; and the time-reversed log-likelihood process

$$
\log \ell(T-s, \widehat{X}(s)), \quad 0 \leq s \leq T \quad \text { is a }(\widehat{\mathbb{G}}, \mathbb{P}) \text { - submartingale. }
$$

Proof: The first claim follows from (5.8) and the convexity of the function $\Phi(\ell)=\ell \log \ell$ appearing inside the expectation in (7.4), along with the JENSEN inequality. The $\mathbb{Q}$-expectation

$$
H(P(T-s) \mid Q)=\mathbb{E}^{\mathbb{Q}}[\Phi(\ell(T-s, \widehat{X}(s)))], \quad 0 \leq s \leq T
$$

of the process in (7.6) is thus increasing. This is precisely the monotonicity in (7.3); the remaining claim

$$
\lim _{t \rightarrow \infty} \downarrow H(P(t) \mid Q)=0
$$

there, follows now from (3.6), (7.4), and the finiteness of $\mathcal{S}$. The claim of (7.7) is a consequence of (7.6), (5.4), and the familiar BAYES rule (Lemma 3.5.3 in KARATZAS \& SHREve (1988)). 


\subsection{Trajectorial Relative Entropy Dissipation}

We read now Proposition 5.1 with $\Phi(\ell)=\ell \log \ell$ and the function

$$
h(s, x)=\Phi(\ell(T-s, x)), \quad 0 \leq s \leq T, x \in \mathcal{S} .
$$

As argued in Propositions 5.2 and 7.1, the "time-reversed relative entropy" $H(P(T-s) \mid Q)=\mathbb{E}^{\mathbb{Q}}[h(s, \widehat{X}(s))]$, $0 \leq s \leq T$ is increasing; and

$$
\widehat{M}^{h}(s):=h(s, \widehat{X}(s))-\int_{0}^{s}(\partial h+\widehat{\mathcal{K}} h)(u, \widehat{X}(u)) \mathrm{d} u, \quad 0 \leq s \leq T
$$

is a $\mathbb{Q}$-local-martingale of the time-reversed filtration $\widehat{\mathbb{G}}$. The integrand in (7.11) is straightforward to compute: from (3.9), (3.10), and with $t=T-s$ for notational convenience, we get

$$
\begin{array}{r}
\partial h(s, x)=-(1+\log \ell(t, x))(\widehat{\mathcal{K}} \ell)(t, x)=-(1+\log \ell(t, x)) \sum_{y \in \mathcal{S}} \widehat{\kappa}(x, y) \ell(t, y), \quad \text { thus } \\
(\partial h+\widehat{\mathcal{K}} h)(s, x)=\sum_{y \in \mathcal{S}} \widehat{\kappa}(x, y) \ell(t, y)\left[\log \frac{\ell(t, y)}{\ell(t, x)}-1\right]=\ell(t, x) \sum_{\substack{y \in \mathcal{S} \\
y \neq x}} \widehat{\kappa}(x, y) \Psi\left(\frac{\ell(t, y)}{\ell(t, x)}\right) \geq 0 .
\end{array}
$$

Here the function

$$
\Psi(r):=r \log r-r+1, \quad r>0
$$

is nonnegative, convex, and attains its minimum $\Psi(1)=0$ at $r=1$. We have used in the last equality of (7.12) the property $\sum_{y \in \mathcal{S}} \widehat{\kappa}(x, y)=0$ for every $x \in \mathcal{S}$.

Proposition 7.2. The submartingales of (7.6), (7.7) admit the respective DOOB-MEYER decompositions

$$
\begin{gathered}
\ell(T-s, \widehat{X}(s)) \log (\ell(T-s, \widehat{X}(s)))=\widehat{M}^{h}(s)+\int_{0}^{s} \lambda^{\mathbb{Q}}(u) \mathrm{d} u, \quad 0 \leq s \leq T, \\
\log (\ell(T-s, \widehat{X}(s)))=\widehat{N}^{h}(s)+\int_{0}^{s} \lambda^{\mathbb{P}}(u) \mathrm{d} u, \quad 0 \leq s \leq T,
\end{gathered}
$$

in the notation of (7.12), (7.13), with $\lambda^{\mathbb{Q}}(s)=\Lambda^{\mathbb{Q}}(T-s, \widehat{X}(s)), \lambda^{\mathbb{P}}(s)=\Lambda^{\mathbb{P}}(T-s, \widehat{X}(s))$ and

$$
\Lambda^{\mathbb{P}}(t, x):=\sum_{y \in \mathcal{S}, y \neq x} \widehat{\kappa}(x, y) \Psi\left(\frac{\ell(t, y)}{\ell(t, x)}\right) \geq 0, \quad \Lambda^{\mathbb{Q}}(t, x):=\ell(t, x) \Lambda^{\mathbb{P}}(t, x) \geq 0 .
$$

Here $\widehat{M}^{h}$ is the process of $(7.11)$ and $a(\widehat{\mathbb{G}}, \mathbb{Q})$-martingale, whereas $\widehat{N}^{h}$ is a $(\widehat{\mathbb{G}}, \mathbb{P})$-martingale.

Proof: Let us take a look at the expressions of (7.10)-(7.12). We have already noted that each function $[0, T] \ni t \mapsto p(t, x) \in(0,1)$ is uniformly continuous. This fact, along with the finiteness of the state space $\mathcal{S}$, implies that the quantities in (7.10), (7.12) are actually uniformly bounded. This implies a similar boundedness for the $(\widehat{\mathbb{G}}, \mathbb{Q})$-local martingale in $(7.11)$, which is thus seen to be a true $(\widehat{\mathbb{G}}, \mathbb{Q})$ - martingale. The remaining claims follow from the BAYES rule.

The decomposition (7.14) is a trajectorial version of relative entropy dissipation. This manifests itself at the level of the individual particles that undergo the MARKOV Chain motion viewed under the lens of the probability measure $\mathbb{Q}$ and under time-reversal, rather than only at the level of their ensembles. 
We note that the first quantity of (7.16) provides the exact rate of relative entropy dissipation, in the sense that for every $0<t<T<\infty$ we have the following convergence, a.e. and in $\mathbb{L}^{1}(\mathbb{P})$ :

$$
\lim _{s \uparrow T-t} \frac{1}{T-t-s}\left(\mathbb{E}^{\mathbb{P}}[\log \ell(t, X(t)) \mid \widehat{\mathcal{G}}(s)]-\log (\ell(T-s, \widehat{X}(s)))\right)=\Lambda^{\mathbb{P}}(t, X(t)) .
$$

The decomposition (7.15) and the trajectorial rate (7.17) are exact analogues of those in Theorem 4.1 and Proposition 4.5 of KARATZAS, SCHACHERMAYER \& TSCHIDERER (2020). They constitute trajectorial versions of relative entropy dissipation, viewed now under the original probability measure $\mathbb{P}$ - and again under time-reversal.

\subsection{DE BRUIJN-Type Identities}

With this preparation, we are now in a position to recover the precise rate of decay for the relative entropy function in (7.2); cf. DiaCONIS \& SALOFF-Coste (1996), Lemma 2.5. All this takes, is to "aggregate" in (7.14) by taking $\mathbb{Q}$-expectations. This leads to an analogue of equation (4.14) in KARATZAS, SCHACHERMAYER \& TSCHIDERER (2020), as we describe now. ${ }^{1}$

Theorem 7.3. DE BRUIJN-type identity for the Dissipation of Relative Entropy: The relative entropy of (7.2) is a decreasing function of time, and satisfies

$$
H(P(T) \mid Q)=H(P(0) \mid Q)-\int_{0}^{T} I(t) \mathrm{d} t=\int_{T}^{\infty} I(t) \mathrm{d} t, \quad I(t)=\mathcal{E}\left(\ell_{t}, \log \boldsymbol{\ell}_{t}\right) \geq 0
$$

for all $T \in[0, \infty)$, in the notation of (4.8), (3.8).

Proof: The first claim is simply a restatement of (7.3); and by taking $\mathbb{Q}$-expectations in (7.14) we obtain in conjunction with (7.10) the first equality of (7.18), with

$$
I(t):=\mathbb{E}^{\mathbb{Q}}[(\partial h+\widehat{\mathcal{K}} h)(T-t, X(t))] .
$$

From (7.12) and (4.8), this quantity coincides with the last expression in (7.18): to wit,

$$
\begin{gathered}
I(t)=\sum_{x \in \mathcal{S}} q(x)(\partial h+\widehat{\mathcal{K}} h)(T-t, x)=\sum_{(x, y) \in \mathcal{Z}} q(x) \widehat{\kappa}(x, y) \ell(t, y)\left[\log \frac{\ell(t, y)}{\ell(t, x)}-1\right] \\
=\sum_{x \in \mathcal{S}} q(x) \ell(t, x) \sum_{\substack{y \in \mathcal{S} \\
y \neq x}} \widehat{\kappa}(x, y) \Psi\left(\frac{\ell(t, y)}{\ell(t, x)}\right)=-\sum_{x \in \mathcal{S}} \sum_{y \in \mathcal{S}} \kappa(y, x) q(y) \ell(t, y) \log \ell(t, x)=\mathcal{E}\left(\ell_{t}, \log \boldsymbol{\ell}_{t}\right) .
\end{gathered}
$$

It is non-negative on account of the non-negativity of the last expression in (7.12), and uniformly continuous as a function of time. In the display (7.19), the second equality follows from the first equality in (7.12); the third from the last equality in (7.12); the fourth from (3.10) and the property $\sum_{y \in \mathcal{S}} \kappa(y, x)=0$ for every $y \in \mathcal{S}$; and the fifth from the definition (4.8). We deduce $H(P(0) \mid Q)=\int_{0}^{\infty} I(t) \mathrm{d} t$ by letting $T \rightarrow \infty$ in (7.18) and recalling (7.9); then the second identity in (7.18) follows.

\footnotetext{
1 The seminal paper STAM (1959), from the early days of Information Theory, establishes the first identity of this type, and in a context where the invariant measure $Q$ is standard Gaussian. A.J. STAM gives credit for this result to his teacher, the analyst, number theorist, combinatorialist and logician Nicolaas DE BRUIJN.
} 
Remark 7.1. Whenever there exists a positive real constant $\alpha$ (respectively, $\beta$ ) such that the POINCARÉ (resp., the modified log-SoBOLEV) inequality

$$
\alpha \leq \frac{2 \mathcal{E}(f, f)}{\sum_{y \in \mathcal{S}} q(y) f^{2}(y)-1} \quad\left(\text { resp., } \quad \beta \leq \frac{\mathcal{E}(f, \log f)}{\sum_{y \in \mathcal{S}} q(y) f(y) \log f(y)}\right)
$$

holds for every function $f: \mathcal{S} \rightarrow(0, \infty)$ with $\sum_{y \in \mathcal{S}} q(y) f(y)=1$, it is clear from (6.5), (6.6) and (7.4), (7.18) that the variance (resp., the relative entropy) decays exponentially:

$$
\operatorname{Var}^{\mathbb{Q}}(L(t)) \leq \operatorname{Var}^{\mathbb{Q}}(L(0)) e^{-\alpha t} \quad\left(\text { resp., } H(P(t) \mid Q) \leq H(P(0) \mid Q) e^{-\beta t}\right) .
$$

Remark 7.2. Expressions for entropy dissipation in a MARKOV Chain context appear in, e.g., MiCLO (1992), Lemma 2.5 of Diaconis \& Saloff-Coste (1996), Bobkov \& Tetali (2006), MonteneGRO \& TETALi (2006), CAPUTO ET AL. (2009) and CONFORTI (2020). These authors use slightly different arguments, based on semigroups. One advantage of the more probabilistic approach we follow here, is that it provides a very sharp picture for the dissipation of relative entropy along trajectories, as exemplified in subsection 7.1.

\subsection{FISHER Information Under Detailed Balance}

The following is now a direct consequence of Lemma 4.2.

Proposition 7.4. Under the detailed-balance condition (3.11), the rate of relative entropy dissipation in (7.18) can be cast as

$$
\begin{aligned}
I(t)=\mathcal{E}\left(\ell_{t}, \log \boldsymbol{\ell}_{t}\right) & =\frac{1}{2} \sum_{(x, y) \in \mathcal{Z}}(\log \ell(t, y)-\log \ell(t, x))^{2} \Theta(\ell(t, y), \ell(t, x)) \kappa(y, x) q(y) \\
& =\frac{1}{2} \sum_{(x, y) \in \mathcal{Z}} \frac{(\ell(t, y)-\ell(t, x))^{2}}{\Theta(\ell(t, y), \ell(t, x))} \kappa(y, x) q(y)
\end{aligned}
$$

in terms of the "logarithmic mean" function

$$
\Theta(q, p):=\frac{q-p}{\log q-\log p}=\int_{0}^{1} q^{r} p^{1-r} \mathrm{~d} r, \quad(q, p) \in(0, \infty)^{2} .
$$

Remark 7.3. The expression in (7.22) is reminiscent of the familiar FISHER Information in Statistics and Information Theory; cf. BOBKOV \& TETALI (2006). Always under the detailed-balance condition (3.11), the expression of (7.22) can be expressed in terms of a "score function", the discrete logarithmic-gradient of the likelihood ratio, as $\left\langle\nabla \ell_{t}, \nabla \log \boldsymbol{\ell}_{t}\right\rangle_{\mathbb{L}^{2}(\mathcal{Z}, C)}$ in the notation of (4.1)-(4.5).

As shown in BOBKOV \& TETALI (2006), the inequality $2(a-b)^{2} \leq\left(a^{2}-b^{2}\right) \log (a / b)$ for $0<a, b<$ $\infty$ leads under detailed-balance (3.11) to the DiACONIS AND SALOFF-CosTE (1996) estimate

$$
\mathcal{E}\left(e^{g}, g\right) \geq 4 \mathcal{E}\left(e^{g / 2}, e^{g / 2}\right), \quad \text { and thus to } \quad I(t)=\mathcal{E}\left(\boldsymbol{\ell}_{t}, \log \boldsymbol{\ell}_{t}\right) \geq 4 \mathcal{E}\left(\sqrt{\boldsymbol{\ell}_{t}}, \sqrt{\boldsymbol{\ell}_{t}}\right) .
$$




\section{The $\Phi$-Relative Entropy Process}

In order to reveal the common thread running through the examples of the last two Sections, let us consider now a continuously differentiable and convex function $\Phi:(0, \infty) \rightarrow \mathbb{R}$ with $\Phi(1)=0$ with continuous, strictly positive second derivative. We denote by $\varphi:(0, \infty) \rightarrow \mathbb{R}$ its derivative $\Phi^{\prime}=\varphi$. For each $\eta>0$, $\xi>0$ we define the BREGMAN $\Phi$-divergence

$$
\operatorname{div}^{\Phi}(\eta \mid \xi):=\Phi(\eta)-\Phi(\xi)-(\eta-\xi) \varphi(\xi),
$$

a quantity which is non-negative on account of the convexity of $\Phi$ (and has nothing to do with the "discrete divergence" we introduced in (4.2)). For instance, $\operatorname{div}^{\Phi}(\eta \mid \xi)=(\xi-\eta)^{2}$ for $\Phi(\xi)=\xi^{2}-1$; whereas, for $\Phi(\xi)=\xi \log \xi$ and in the notation of (7.13), we have

$$
\operatorname{div}^{\Phi}(\eta \mid \xi)=\operatorname{div}^{\Psi}(\eta \mid \xi)=\xi \Psi(\eta / \xi) .
$$

Let us consider now, for a general convex $\Phi$ as above, the so-called $\Phi$-relative entropy

$$
H^{\Phi}(P(t) \mid Q):=\mathbb{E}^{\mathbb{Q}}\left[\Phi(\ell(t, X(t))]=\sum_{y \in \mathcal{S}} q(y) \Phi\left(\frac{p(t, y)}{q(y)}\right), \quad 0 \leq t<\infty ;\right.
$$

see CHAFAï (2004) for a general study of such functions. The convexity of $\Phi$ and the JENSEN inequality imply that this function is nonnegative, since $\Phi(1)=0$; and from Proposition 5.2, that the time-reversed $\Phi-$ relative entropy process

$$
\Phi(\ell(T-s, \widehat{X}(s)), \quad 0 \leq s \leq T
$$

is a $(\widehat{\mathbb{G}}, \mathbb{Q})$-submartingale, for every fixed $T \in(0, \infty)$. As a consequence the function in (8.3) decreases, in fact satisfies $\lim _{t \rightarrow \infty} \downarrow H^{\Phi}(P(t) \mid Q)=0$ by virtue of (3.6) and the finiteness of the state space.

\subsection{Trajectorial Dissipation of the $\Phi-$ Relative Entropy}

The Doob-Meyer decomposition of this submartingale is obtained from Proposition 5.1 as follows: Consider the function $g(s, x)=\Phi(\ell(T-s, x))$ and compute, in the manner of (7.12), the quantities

$\partial g(s, x)=-\varphi(\ell(t, x)) \sum_{y \in \mathcal{S}} \widehat{\kappa}(x, y)[\ell(t, y)-\ell(t, x)], \quad(\widehat{\mathcal{K}} g)(s, x)=\sum_{y \in \mathcal{S}} \widehat{\kappa}(x, y)[\Phi(\ell(t, y))-\Phi(\ell(t, x))]$ with $t=T-s$, on account of (3.9). Putting these expressions together with (8.1), we deduce

$$
(\partial g+\widehat{\mathcal{K}} g)(s, x)=\left.\sum_{y \in \mathcal{S}, y \neq x} \widehat{\kappa}(x, y) \operatorname{div}^{\Phi}(\eta \mid \xi)\right|_{\substack{\eta=\ell(t, y) \\ \xi=\ell(t, x)}}=: \Lambda^{\Phi, \mathbb{Q}}(t, x) \geq 0 .
$$

The following result is now a direct consequence of Proposition 5.1 and the BAYES rule. Once again, the finiteness of the state-space and the continuity of the functions involved, turn local into true martingales.

Proposition 8.1. For any given $T \in(0, \infty)$, the process below is a $(\widehat{\mathbb{G}}, \mathbb{Q})$-martingale:

$$
\Phi(\ell(T-s, \widehat{X}(s)))-\int_{0}^{s} \Lambda^{\Phi, \mathbb{Q}}(T-u, \widehat{X}(u)) \mathrm{d} u, \quad 0 \leq s \leq T .
$$

Whereas, with $\Lambda^{\Phi, \mathbb{P}}(t, x):=\Lambda^{\Phi, \mathbb{Q}}(t, x) / \ell(t, x)$, the process below is a $(\widehat{\mathbb{G}}, \mathbb{P})$ - martingale:

$$
\frac{\Phi(\ell(T-s, \widehat{X}(s)))}{\ell(T-s, \widehat{X}(s))}-\int_{0}^{s} \Lambda^{\Phi, \mathbb{P}}(T-u, \widehat{X}(u)) \mathrm{d} u, \quad 0 \leq s \leq T .
$$




\subsection{Generalized DE BRUIJN Identities}

In view of these considerations, it is now straightforward to "aggregate" (i.e., take $\mathbb{Q}$-expectations of) the $(\widehat{\mathbb{G}}, \mathbb{Q})$ - martingale of $(8.5)$. We obtain in the manner of $(7.18)$ the following result, stated again in the forward direction of time; cf. CHAFAÏ (2004), Proposition 1.1.

Proposition 8.2. Generalized DE BRUIJN-type identity: The temporal dissipation of the $\Phi$-relative entropy of (8.3) is given for $0 \leq T<\infty$ as

$H^{\Phi}(P(T) \mid Q)=H^{\Phi}(P(0) \mid Q)-\int_{0}^{T} I^{\Phi}(t) \mathrm{d} t=\int_{T}^{\infty} I^{\Phi}(t) \mathrm{d} t, \quad I^{\Phi}(t):=\mathbb{E}^{\mathbb{Q}}\left[\Lambda^{\Phi, \mathbb{Q}}(t, X(t))\right] \geq 0$.

On the strength of (8.4), the dissipation rate in (8.7) is given by the $\Phi-$ FISHER Information

$$
\begin{gathered}
I^{\Phi}(t)=\sum_{(x, y) \in \mathcal{Z}} q(x) \widehat{\kappa}(x, y) \operatorname{div}^{\Phi}(\ell(t, y) \mid \ell(t, x))=\sum_{x \in \mathcal{S}} \sum_{y \in \mathcal{S}} q(x) \widehat{\kappa}(x, y) \operatorname{div}^{\Phi}(\ell(t, y) \mid \ell(t, x)) \\
=-\sum_{x \in \mathcal{S}} \sum_{y \in \mathcal{S}} q(y) \kappa(y, x) \ell(t, y) \varphi(\ell(t, x))=\mathcal{E}\left(\boldsymbol{\ell}_{t}, \varphi\left(\boldsymbol{\ell}_{t}\right)\right) .
\end{gathered}
$$

Proof: The third equality in (8.8) is a consequence of the properties $\sum_{y \in \mathcal{S}} \widehat{\kappa}(x, y)=0$ for every $x \in \mathcal{S}$, and $\sum_{x \in \mathcal{S}} \kappa(y, x)=0$ for every $y \in \mathcal{S}$, as well as of (3.10). It underscores the fact that, when passing from the trajectorial to the "aggregate" point of view (that is, when taking $\mathbb{Q}$-expectations), the term $\xi \varphi(\xi)-\Phi(\xi)$ that depends only on the variable $\xi=\ell(t, x)$, as well as the term $\Phi(\eta)$ that depends only on the variable $\eta=\ell(t, y)$, can be ignored in (8.1); only the "mixed term" $-\eta \varphi(\xi)$ remains relevant. We note that similar reasoning was deployed in the proof of Lemma 4.1.

Remark 8.1. Some Special Cases: ( $i$ ) For the convex function $\Phi(\xi)=\xi \log \xi$, and recalling (8.2), (7.13), the quantity $I^{\Phi}(t)$ of (8.8) is seen to coincide with $I(t)$ in (7.19), (7.18).

(ii) On the other hand, when $\Phi(\xi)=\xi^{2}-1$ we have $\operatorname{div}^{\Phi}(\eta \mid \xi)=(\eta-\xi)^{2}$ in (8.1) and

$H^{\Phi}(P(t) \mid Q)=\mathbb{E}^{\mathbb{Q}}\left(\ell^{2}(t, X(t))-1=\left|\ell_{t}\right|_{\mathbb{L}^{2}(\mathcal{S}, Q)}^{2}-1=\operatorname{Var}^{\mathbb{Q}}(L(t))=V(P(t) \mid Q), \quad 0 \leq t<\infty\right.$

as in (6.1), and the rate of temporal dissipation for this function is precisely the integrand in (6.8):

$$
I^{\Phi}(t)=-2 \sum_{x \in \mathcal{S}} \sum_{y \in \mathcal{S}} q(y) \kappa(y, x) \ell(t, x) \ell(t, y)=2 \mathcal{E}\left(\ell_{t}, \ell_{t}\right) .
$$

(iii) A bit more generally, the choice of convex function $\Phi(\xi)=\left(\xi^{m}-1\right) /(m-1)$ with $m>1$, leads to the so-called "RÉNYI relative entropy"

$$
H^{\Phi}(P(t) \mid Q)=\frac{\mathbb{E}^{\mathbb{Q}}\left(\ell^{m}(t, X(t))-1\right.}{m-1}, \quad 0 \leq t<\infty
$$

whose rate of temporal dissipation is a generalized version of (8.9):

$$
I^{\Phi}(t)=-\frac{m}{m-1} \sum_{x \in \mathcal{S}} \sum_{y \in \mathcal{S}} q(y) \kappa(y, x) \ell(t, y)(\ell(t, x))^{m-1}=\frac{m}{m-1} \mathcal{E}\left(\boldsymbol{\ell}_{t}, \boldsymbol{\ell}_{t}^{m-1}\right) .
$$

The variance $\operatorname{Var}^{\mathbb{Q}}(L(t))$ is thus a special case of the RÉNYI relative entropy, corresponding to $m=2$; whereas the relative entropy in (7.4) corresponds to the limit of (8.10) as $m \downarrow 1$.

We stress that nowhere in this subsection, or in the one preceding it, did we invoke the detailed-balance conditions of (3.11). 


\subsection{Locally Steepest Descent for the $\Phi$-Relative Entropy Under Detailed Balance}

We formulate now a variational version of Proposition 8.2 under the conditions (3.11) of detailed balance. These will be in force throughout the current subsection.

Remark 8.2. First, let us take a look at the expression of (8.8). From the consequence $q(x) \widehat{\kappa}(x, y)=$ $q(y) \kappa(y, x)=q(y) \widehat{\kappa}(y, x)$ of the detailed balance conditions (3.11), as well as from the consequence

$$
\operatorname{div}^{\Phi}(\eta \mid \xi)+\operatorname{div}^{\Phi}(\xi \mid \eta)=(\eta-\xi)(\varphi(\eta)-\varphi(\xi))
$$

of (8.1), we see that the $\Phi-$ FISHER Information of (8.8) can be cast in this case as

$$
\begin{aligned}
& I^{\Phi}(t)=\left.\frac{1}{2} \sum_{x \in \mathcal{S}} \sum_{y \in \mathcal{S}} q(x) \widehat{\kappa}(x, y)\left(\operatorname{div}^{\Phi}(\eta \mid \xi)+\operatorname{div}^{\Phi}(\xi \mid \eta)\right)\right|_{\substack{\eta=\ell(t, y) \\
\xi=\ell(t, x)}} \\
& =\left.\frac{1}{2} \sum_{x \in \mathcal{S}} \sum_{y \in \mathcal{S}} q(y) \kappa(y, x)((\eta-\xi)(\varphi(\eta)-\varphi(\xi)))\right|_{\substack{\eta=\ell(t, y) \\
\xi=\ell(t, x)}}=\mathcal{E}\left(\varphi\left(\boldsymbol{\ell}_{t}\right), \boldsymbol{\ell}_{t}\right) \\
& =\left.\frac{1}{2} \sum_{(x, y) \in \mathcal{Z}} q(x) \kappa(x, y) \Theta^{\Phi}(\xi, \eta)(\varphi(\xi)-\varphi(\eta))^{2}\right|_{\substack{\xi=\ell(t, x) \\
\eta=\ell(t, y)}}
\end{aligned}
$$

in the manner of (7.22); we recall the notation $\varphi=\Phi^{\prime}$. Here, the function

$$
\Theta^{\Phi}(q, p):=\frac{q-p}{\varphi(q)-\varphi(p)}, \quad 0<q \neq p<\infty, \quad \Theta^{\Phi}(p, p):=\frac{1}{\Phi^{\prime \prime}(p)}, \quad 0<p<\infty,
$$

extends the "logarithmic mean" of (7.23), to which it reduces when $\Phi(\xi)=\xi \log \xi$. With $\Phi(\xi)=\xi^{2}-1$ we get $\Theta^{\Phi} \equiv 1 / 2$, and the last expression in (8.11) reduces to $\sum_{(x, y) \in \mathcal{Z}} q(x) \kappa(x, y) \cdot(\ell(t, x)-\ell(t, y))^{2}$ as in (6.8). We shall comment further on this choice of (8.12), in subsection 9.2 below.

We set out now to find a metric on the manifold $\mathcal{M}=\mathcal{P}_{+}(\mathcal{S})$ of probability vectors on the state-space, relative to which the time-marginals for the MARKOV Chain $(P(t))_{0 \leq t<\infty}$ constitute a curve of steepest descent for the $\Phi$-relative entropy. In other words, we look for a metric on $\mathcal{M}$ that can play - in the current general context - a role similar to that played by the HILBERT norm $\|\cdot\|_{\mathbb{H}^{-1}(\mathcal{S}, Q)}$ in Section 6.

This norm defines the metric distance of (6.10) that works for the variance $V(P(t) \mid Q)$, i.e., in the special case $\Phi(\xi)=\xi^{2}-1$. But except for such very special cases, the Riemannian metric on the manifold $\mathcal{M}$ will not be flat; i.e., not induced by such a simple norm as in Proposition 6.5. For this reason we are forced to consider the machinery of Riemannian geometry, which we take up in the next Section 9. In this Section we avoid Riemannian terminology, and present the steepest descent property of the curve $(P(t))_{0 \leq t<\infty}$ in terms of appropriate HILBERT norms that capture the local behavior of the Riemannian metric.

\subsubsection{Locally Weighted SoboLEV Norms}

We start this effort by recalling from (4.6) the norm $\|F\|_{\mathbb{L}^{2}(\mathcal{Z}, C)}$ for functions $F: \mathcal{Z} \rightarrow \mathbb{R}$. This is defined on the "off-diagonal Cartesian product" $\mathcal{Z}$ by assigning to its elements $(x, y)$, where $x \neq y$, the weights $c(x, y)=q(x) \kappa(x, y) / 2$ and taking the usual $\mathbb{L}^{2}$-norm relative to the positive measure with these weights. For a fixed likelihood ratio $\ell$ in the space $\mathcal{L}=\mathcal{L}_{+}(\mathcal{S})$ of subsection 3.2 we consider now, in place of $c(x, y) \equiv q(x) \kappa(x, y) / 2$ and with the notation of (8.12), the new weights

$$
c(x, y) \cdot \vartheta_{\ell}(x, y), \quad \text { where } \quad \vartheta_{\ell}(x, y):=\Theta^{\Phi}(\ell(x), \ell(y))=\frac{\nabla \ell(x, y)}{\nabla(\varphi \circ \ell)(x, y)} .
$$


The resulting weighted inner product and norm, extensions of the respective quantities for real-valued functions on $\mathcal{S} \times \mathcal{S}$ in (4.5), (4.6) (to which they reduce when $\Phi(\xi)=\xi^{2} / 2$ ), are respectively

$$
\begin{aligned}
\langle F, G\rangle_{\mathbb{L}^{2}\left(\mathcal{Z}, \vartheta_{\ell} C\right)} & :=\sum_{(x, y) \in \mathcal{Z}} c(x, y) \vartheta_{\ell}(x, y) F(x, y) G(x, y)=\left\langle\vartheta_{\ell} F, G\right\rangle_{\mathbb{L}^{2}(\mathcal{Z}, C)}, \\
\|F\|_{\mathbb{L}^{2}\left(\mathcal{Z}, \vartheta_{\ell} C\right)}^{2} & :=\langle F, F\rangle_{\mathbb{L}^{2}\left(\mathcal{Z}, \vartheta_{\ell} C\right)} .
\end{aligned}
$$

We define now for $f: \mathcal{S} \rightarrow \mathbb{R}$ the Weighted Sobolev Norm $\|\cdot\|_{\mathbb{H}_{\Theta}^{1}(\mathcal{S}, \ell Q)}$, by replacing on the right-hand sides of (4.11)-(4.13) the norm $\|\cdot\|_{\mathbb{L}^{2}(\mathcal{Z}, C)}$ by the new norm $\|\cdot\|_{\mathbb{L}^{2}\left(\mathcal{Z}, \vartheta_{\ell} C\right)}$ in (8.14):

$$
\langle f, g\rangle_{\mathbb{H}_{\Theta}^{1}(\mathcal{S}, \ell Q)}:=\langle\nabla f, \nabla g\rangle_{\mathbb{L}^{2}\left(\mathcal{Z}, \vartheta_{\ell} C\right)}, \quad\|f\|_{\mathbb{H}_{\Theta}^{1}(\mathcal{S}, \ell Q)}^{2}:=\langle f, f\rangle_{\mathbb{H}_{\Theta}^{1}(\mathcal{S}, \ell Q)}=\|\nabla f\|_{\mathbb{L}^{2}\left(\mathcal{Z}, \vartheta_{\ell} C\right)}^{2} .
$$

Remark 8.3. It is interesting to note at this point, and will become quite important down the road, that the $\Phi$-FISHER Information of (8.8), (8.11) can be expressed in terms of the square of this new, weighted SOBOLEV norm. Indeed, for any $\ell \in \mathcal{L}_{+}(\mathcal{S})$ we have

$$
\mathcal{E}(\ell, \varphi(\ell))=\langle\nabla \ell, \nabla \varphi(\ell)\rangle_{\mathbb{L}^{2}(\mathcal{Z}, C)}=\left\langle\vartheta_{\ell} \nabla \varphi(\ell), \nabla \varphi(\ell)\right\rangle_{\mathbb{L}^{2}(\mathcal{Z}, C)}=\|\nabla \varphi(\ell)\|_{\mathbb{L}^{2}\left(\mathcal{Z}, \vartheta_{\ell} C\right)}^{2}=\|\varphi(\ell)\|_{\mathbb{H}_{\Theta}^{1}(\mathcal{S}, \ell Q)}^{2} .
$$

Thus, the $\Phi$-FISHER Information of (8.8) takes the form $I^{\Phi}(t)=\left\|\varphi\left(\ell_{t}\right)\right\|_{\mathbb{H}_{\Theta}^{1}\left(\mathcal{S}, \ell_{t} Q\right)}^{2}$.

Finally, we introduce in the manner of (4.14), (4.15) the dual of this weighted SOBOLEV norm

$$
\|f\|_{\mathbb{H}_{\Theta}^{-1}(\mathcal{S}, \ell Q)}:=\sup _{g: \mathcal{S} \rightarrow \mathbb{R}} \frac{\langle f, g\rangle_{\mathbb{L}^{2}(\mathcal{S}, Q)}}{\|g\|_{\mathbb{H}_{\Theta}^{1}(\mathcal{S}, \ell Q)}} .
$$

This admits a variational characterization analogous to (4.15), which will be crucial in what follows.

Proposition 8.3. Variational Interpretation: For any function $f: \mathcal{S} \rightarrow \mathbb{R}$ we have

$$
\|f\|_{\mathbb{H}_{\Theta}^{-1}(\mathcal{S}, \ell Q)}=\inf _{G: \mathcal{Z} \rightarrow \mathbb{R}}\left\{\|G\|_{\mathbb{L}^{2}\left(\mathcal{Z}, \vartheta_{\ell} C\right)}: f+\nabla \cdot\left(\vartheta_{\ell} G\right)=0\right\} .
$$

Moreover, $\|f\|_{\mathbb{H}_{\Theta}^{-1}(\mathcal{S}, \ell Q)}$ is finite if, and only if, $\sum_{x \in \mathcal{S}} q(x) f(x)=0$; in this case the infimum is attained, and uniquely, by the unique discrete gradient that is admissible.

Proof. Consider a function $f: \mathcal{S} \rightarrow \mathbb{R}$ such that $\sum_{x \in \mathcal{S}} q(x) f(x)=0$; if this is not the case, it is straightforward to verify that both sides in (8.17) are infinite. We note that the set of admissible $G$ on the right-hand side of (8.17) is non-empty (indeed, $G_{0}:=-\frac{1}{\vartheta_{\ell}} \nabla \mathcal{K}^{-1} f$ is admissible) and that a minimizer exists.

Let $G: \mathcal{Z} \rightarrow \mathbb{R}$ be such a minimizer. We show first that $G$ is a discrete gradient, by a projection argument in the HILBERT space $\mathbb{L}^{2}\left(\mathcal{Z}, \vartheta_{\ell} C\right)$.

To this end, let us denote by $\nabla h$ the orthogonal projection of $G$ onto the subspace of discrete gradients in $\mathbb{L}^{2}\left(\mathcal{Z}, \vartheta_{\ell} C\right)$. We claim that $\nabla h$ is admissible on the right-hand side of (8.17). Indeed, $G-\nabla h$ is orthogonal in $\mathbb{L}^{2}\left(\mathcal{Z}, \vartheta_{\ell} C\right)$ to $\nabla g$ for any $g: \mathcal{S} \rightarrow \mathbb{R}$. This implies

$$
-\left\langle g, \nabla \cdot\left(\vartheta_{\ell}(G-\nabla h)\right)\right\rangle_{\mathbb{L}^{2}(\mathcal{S}, Q)}=\left\langle\nabla g, \vartheta_{\ell}(G-\nabla h)\right\rangle_{\mathbb{L}^{2}(\mathcal{Z}, C)}=\langle\nabla g, G-\nabla h\rangle_{\mathbb{L}^{2}\left(\mathcal{Z}, \vartheta_{\ell} C\right)}=0
$$

and yields $\nabla \cdot\left(\vartheta_{\ell} G\right)=\nabla \cdot\left(\vartheta_{\ell} \nabla h\right)$, proving the claim.

By orthogonality, we have $\|G\|_{\mathbb{L}^{2}\left(\mathcal{Z}, \vartheta_{\ell} C\right)}^{2}=\|\nabla h\|_{\mathbb{L}^{2}\left(\mathcal{Z}, \vartheta_{\ell} C\right)}^{2}+\|G-\nabla h\|_{\mathbb{L}^{2}\left(\mathcal{Z}, \vartheta_{\ell} C\right)}^{2}$. Since $G$ is a minimizer, we infer $\|G-\nabla h\|_{\mathbb{L}^{2}\left(\mathcal{Z}, \vartheta_{\ell} C\right)}=0$, which implies that $G \equiv \nabla h$. This shows that $\nabla h$ is a minimizer, 
and that the right-hand side of (8.17) is equal to $\|h\|_{\mathbb{H}_{\Theta}^{1}(\mathcal{S}, \ell Q)}$. It is shown in MAAS (2011) that $\nabla h$ is actually the unique discrete gradient satisfying the constraint in (8.17).

To prove the equality in (8.17), we note for any $g: \mathcal{S} \rightarrow \mathbb{R}$ the identities

$$
\langle f, g\rangle_{\mathbb{L}^{2}(\mathcal{S}, Q)}=-\left\langle\nabla \cdot\left(\vartheta_{\ell} \nabla h\right), g\right\rangle_{\mathbb{L}^{2}(\mathcal{S}, Q)}=\left\langle\vartheta_{\ell} \nabla h, \nabla g\right\rangle_{\mathbb{L}^{2}(\mathcal{Z}, C)}=\langle h, g\rangle_{\mathbb{H}_{\Theta}^{1}(\mathcal{S}, \ell Q)}
$$

Writing the dual norm as a LEGENDRE transform, we obtain

$$
\begin{aligned}
\|f\|_{\mathbb{H}_{\Theta}^{-1}(\mathcal{S}, \ell Q)}^{2} & =\sup _{g: \mathcal{S} \rightarrow \mathbb{R}}\left\{2\langle f, g\rangle_{\mathbb{L}^{2}(\mathcal{S}, Q)}-\|g\|_{\mathbb{H}_{\Theta}^{1}(\mathcal{S}, \ell Q)}^{2}\right\} \\
& =\sup _{g: \mathcal{S} \rightarrow \mathbb{R}}\left\{2\langle h, g\rangle_{\mathbb{H}_{\Theta}^{1}(\mathcal{S}, \ell Q)}-\|g\|_{\mathbb{H}_{\Theta}^{1}(\mathcal{S}, \ell Q)}^{2}\right\}=\|h\|_{\mathbb{H}_{\Theta}^{1}(\mathcal{S}, \ell Q)}^{2},
\end{aligned}
$$

which establishes the equality in (8.17).

Let us consider now as in Section 6, for some $\varepsilon>0$ an arbitrary smooth curve $\ell^{\psi}(\cdot)=\left(\ell^{\psi}(t)\right)_{t_{0} \leq t<t_{0}+\varepsilon}$ with initial position $\ell^{\psi}\left(t_{0}\right)=\ell \equiv \ell\left(t_{0}\right)$ in $\mathcal{L}=\mathcal{L}_{+}(\mathcal{S})$. In order to compute $\mathbb{H}_{\Theta}^{-1}(\mathcal{S}, \ell Q)$-norms, it is natural in view of Proposition 8.3 to write the time-evolution in the manner of a "discrete continuity equation"

$$
\partial \ell_{t}^{\psi}+\nabla \cdot\left(\vartheta_{\ell_{t}} \nabla \psi_{t}\right)=0
$$

as in subsection 6.1 , where $\psi_{t}: \mathcal{S} \rightarrow \mathbb{R}$ is unique up to an additive constant.

We regard here $\psi(\cdot)$ as an input, whose gradient is the velocity vector field that yields the infinitesimal change $\partial \ell_{t}^{\psi}$ of the likelihood ratio flow. In light of (3.9), (8.13) and detailed balance, the original backward equation $\partial \ell_{t}=\widehat{\mathcal{K}} \ell_{t}=\mathcal{K} \ell_{t}=\nabla \cdot\left(\nabla \ell_{t}\right)=\nabla \cdot\left(\vartheta_{\ell_{t}} \nabla \varphi\left(\ell_{t}\right)\right.$ corresponds to $\psi_{t}=-\varphi\left(\ell_{t}\right)$ in this scheme of things.

We define as in (6.12) the corresponding curve $P^{\psi}(\cdot)=\left(P^{\psi}(t)\right)_{t_{0} \leq t<t_{0}+\varepsilon}$ on the manifold $\mathcal{M}=\mathcal{P}_{+}(\mathcal{S})$ of probability vectors on the state-space. We obtain the following generalization of Proposition 8.2.

Proposition 8.4. In the above context, we have

$$
\partial H^{\Phi}\left(P^{\psi}(t) \mid Q\right)=\left\langle\varphi\left(\ell_{t}^{\psi}\right), \psi_{t}\right\rangle_{\mathbb{H}_{\Theta}^{1}\left(\mathcal{S}, \ell_{t} Q\right)} .
$$

Proof. Using the abovementioned discrete continuity equation, a discrete integration by parts, and the definitions of the scalar products, we deduce

$$
\begin{aligned}
\partial H^{\Phi}\left(P^{\psi}(t) \mid Q\right) & =\partial \mathbb{E}^{\mathbb{Q}}\left[\Phi\left(\ell^{\psi}(t, X(t))\right)\right]=-\left\langle\varphi\left(\ell_{t}^{\psi}\right), \nabla \cdot\left(\vartheta_{\ell_{t}} \nabla \psi_{t}\right)\right\rangle_{\mathbb{L}^{2}(\mathcal{S}, Q)} \\
& =\left\langle\nabla \varphi\left(\ell_{t}^{\psi}\right), \vartheta_{\ell_{t}} \nabla \psi_{t}\right\rangle_{\mathbb{L}^{2}(\mathcal{Z}, C)}=\left\langle\nabla \varphi\left(\ell_{t}^{\psi}\right), \nabla \psi_{t}\right\rangle_{\mathbb{L}^{2}\left(\mathcal{Z}, \vartheta_{\ell_{t}} C\right)}=\left\langle\varphi\left(\ell_{t}^{\psi}\right), \psi_{t}\right\rangle_{\mathbb{H}_{\Theta}^{1}\left(\mathcal{S}, \ell_{t} Q\right)}
\end{aligned}
$$

as desired.

With the context and notation just established, and always for $\ell \equiv \ell\left(t_{0}\right)=\left(\ell\left(t_{0}, x\right)\right)_{x \in \mathcal{S}}$, we can formulate the following analogue of Proposition 6.5. This result uses the characterizations of the weighted $\mathbb{H}^{-1}$-norm in (8.16), along with the identity $\mathcal{K} \ell=\nabla \cdot\left(\vartheta_{\ell} \nabla \varphi(\ell)\right)$.

Proposition 8.5. Under the conditions (3.11) of detailed balance, we have, with $\ell^{\psi}\left(t_{0}\right)=\boldsymbol{\ell} \equiv \boldsymbol{\ell}\left(t_{0}\right)$,

$$
\lim _{h \downarrow 0} \frac{1}{h}\left\|\boldsymbol{\ell}_{t_{0}+h}-\boldsymbol{\ell}_{t_{0}}\right\|_{\mathbb{H}_{\Theta}^{-1}(\mathcal{S}, \ell Q)}=\left\|\mathcal{K} \boldsymbol{\ell}_{t_{0}}\right\|_{\mathbb{H}_{\Theta}^{-1}(\mathcal{S}, \ell Q)}=\left\|\varphi\left(\boldsymbol{\ell}_{t_{0}}\right)\right\|_{\mathbb{H}_{\Theta}^{1}(\mathcal{S}, \ell Q)}
$$

and a bit more generally,

$$
\lim _{h \downarrow 0} \frac{1}{h}\left\|\ell_{t_{0}+h}^{\psi}-\ell_{t_{0}}^{\psi}\right\|_{\mathbb{H}_{\Theta}^{-1}(\mathcal{S}, \ell Q)}=\left\|\nabla \cdot\left(\vartheta_{\ell_{t_{0}}} \nabla \psi_{t_{0}}\right)\right\|_{\mathbb{H}_{\Theta}^{-1}(\mathcal{S}, \ell Q)}=\left\|\psi_{t_{0}}\right\|_{\mathbb{H}_{\Theta}^{1}(\mathcal{S}, \ell Q)} .
$$


We pass now to the principal result of the Section. This generalizes Theorem 6.3, to which it reduces when $\Phi(\xi)=\xi^{2}-1$. It is also a direct analogue of Theorem 3.2 in KARATZAS, SCHACHERMAYER \& TSCHIDERER (2020), where a similar steepest-descent for the relative entropy is established for LANGEVIN diffusions, and with distance on the ambient space measured by the quadratic WASSERSTEIN metric. The role of that metric is played now by the locally flat metric defined in (8.21) below.

Theorem 8.6. Steepest Descent for the $\Phi$-Relative Entropy: Under the detailed-balance conditions (3.11), the curve $(P(t))_{t_{0} \leq t<\infty}$ of time-marginal distributions in (3.4) has the property of steepest descent in Definition 6.1 for the $\Phi$-Relative Entropy of (8.3), locally at $t=t_{0}$, and with respect to the distance induced by the "flat metric"

$$
\varrho_{\star}\left(P_{1}, P_{2}\right):=\left\|\ell_{1}-\ell_{2}\right\|_{\mathbb{H}_{\Theta}^{-1}(\mathcal{S}, \ell Q)} \quad \text { for } P_{1}=\ell_{1} Q \text { and } P_{2}=\ell_{2} Q .
$$

Here again we have $\ell \equiv \ell\left(t_{0}\right)$.

Proof: This is proved exactly as in subsection 6.2, with the caveat that the distance-inducing flat metric is now determined "locally", that is, depends on $\left(t_{0}, \ell\right) \equiv\left(t_{0}, \ell\left(t_{0}\right)\right)$ in the weighted norms of (8.14)-(8.16). We go through the argument again, however, in order to highlight the role that these weighted norms play in the present, more general context. From (8.18), and recalling the initial position $\ell^{\psi}\left(t_{0}\right)=\ell\left(t_{0}\right) \in \mathcal{L}$, we obtain

$$
\lim _{h \downarrow 0} \frac{H^{\Phi}\left(P^{\psi}\left(t_{0}+h\right) \mid Q\right)-H^{\Phi}\left(P\left(t_{0}\right) \mid Q\right)}{h}=\left\langle\varphi\left(\ell_{t_{0}}\right), \psi_{t_{0}}\right\rangle_{\mathbb{H}_{\Theta}^{1}(\mathcal{S}, \ell Q)} ;
$$

whereas (8.20) gives

$$
\lim _{h \downarrow 0} \frac{\varrho_{\star}\left(P^{\psi}\left(t_{0}+h\right), P\left(t_{0}\right)\right)}{h}=\left\|\psi_{t_{0}}\right\|_{\mathbb{H}_{\Theta}^{1}(\mathcal{S}, \ell Q)},
$$

thus

$$
\lim _{h \downarrow 0} \frac{H^{\Phi}\left(P^{\psi}\left(t_{0}+h\right) \mid Q\right)-H^{\Phi}\left(P\left(t_{0}\right) \mid Q\right)}{\varrho_{\star}\left(P^{\psi}\left(t_{0}+h\right), P\left(t_{0}\right)\right)}=\left\langle\varphi\left(\ell_{t_{0}}\right), \frac{\psi_{t_{0}}}{\left\|\psi_{t_{0}}\right\|_{\mathbb{H}_{\Theta}^{1}(\mathcal{S}, \ell Q)}}\right\rangle_{\mathbb{H}_{\Theta}^{1}(\mathcal{S}, \ell Q)} .
$$

This is the rate of change for the $\Phi$-relative entropy along the perturbed curve $\left(P^{\psi}(t)\right)_{t_{0} \leq t<t_{0}+\varepsilon}$, as measured on the manifold $\mathcal{M}$ with respect to the distance in (8.21).

On the other hand, we have from (8.8), (8.7) and (8.11), the following observation: Along the original curve of time-marginal distributions $(P(t))_{t_{0} \leq t<\infty}$ for the Chain, corresponding to taking $\psi(\cdot) \equiv \varphi(\ell)(\cdot)$ above, the rate of $\Phi$-relative entropy dissipation measured in terms of the "flat metric" distance traveled on the manifold $\mathcal{M}$, is given as

$$
\lim _{h \downarrow 0} \frac{H^{\Phi}\left(P\left(t_{0}+h\right) \mid Q\right)-H^{\Phi}\left(P\left(t_{0}\right) \mid Q\right)}{\varrho_{\star}\left(P\left(t_{0}+h\right), P\left(t_{0}\right)\right)}=-\left\|\varphi\left(\ell_{t_{0}}\right)\right\|_{\mathbb{H}_{\Theta}^{1}(\mathcal{S}, \ell Q)}<0 .
$$

A simple comparison of the last two displays, via CAUCHY-SCHWARZ, gives the steepest descent property

$$
\begin{gathered}
\lim _{h \downarrow 0} \frac{H^{\Phi}\left(P^{\psi}\left(t_{0}+h\right) \mid Q\right)-H^{\Phi}\left(P\left(t_{0}\right) \mid Q\right)}{\varrho_{\star}\left(P^{\psi}\left(t_{0}+h\right), P\left(t_{0}\right)\right)}-\lim _{h \downarrow 0} \frac{H^{\Phi}\left(P\left(t_{0}+h\right) \mid Q\right)-H^{\Phi}\left(P\left(t_{0}\right) \mid Q\right)}{\varrho_{\star}\left(P\left(t_{0}+h\right), P\left(t_{0}\right)\right)} \\
=\left\|\varphi\left(\ell_{t_{0}}\right)\right\|_{\mathbb{H}_{\Theta}^{1}(\mathcal{S}, \ell Q)}+\left\langle\varphi\left(\ell_{t_{0}}\right), \frac{\psi_{t_{0}}}{\left\|\psi_{t_{0}}\right\|_{\mathbb{H}_{\Theta}^{1}(\mathcal{S}, \ell Q)}}\right\rangle_{\mathbb{H}_{\Theta}^{1}(\mathcal{S}, \ell Q)} \geq 0
\end{gathered}
$$

of the $\Phi$-relative entropy with respect to the distance in (6.10), along the original curve of MARKOV Chain time-marginals. Equality holds if, and only if, $\nabla \psi_{t_{0}}$ is a negative constant multiple of $\nabla \varphi\left(\ell_{t_{0}}\right)$. 


\subsubsection{Non-uniqueness of the Flat Metric}

There exist norms other than $\mathbb{H}_{\Theta}^{-1}(\mathcal{S}, \ell Q)$ of (8.16), for which Theorem 8.6 remains valid; see DIETERT (2015) and Proposition 9.4 below. Here we exhibit an explicit example.

Fix $\ell \in \mathcal{L}_{+}(\mathcal{S})$ and consider the "modified weighted $\mathbb{H}^{-1}$-norm" given by

$$
\|f\|_{\widetilde{\mathbb{H}}_{\Theta}^{-1}(\mathcal{S}, \ell Q)}^{2}:=\left\langle\frac{1}{\vartheta_{\ell}} \nabla\left(\mathcal{K}^{-1} f\right), \nabla\left(\mathcal{K}^{-1} f\right)\right\rangle_{\mathbb{L}^{2}(\mathcal{Z}, C)}
$$

for functions $f: \mathcal{S} \rightarrow \mathbb{R}$ with $\sum_{x \in \mathcal{S}} f(x) q(x)=0$. This norm is never smaller than the original $\mathbb{H}_{\Theta}^{-1}(\mathcal{S}, \ell Q)-$ norm as defined in (8.15); namely,

$$
\|f\|_{\widetilde{\mathbb{H}}_{\Theta}^{-1}(\mathcal{S}, \ell Q)} \geq\|f\|_{\mathbb{H}_{\Theta}^{-1}(\mathcal{S}, \ell Q)} \cdot
$$

And equality holds when $f=\mathcal{K} \ell$; to wit,

$$
\|\mathcal{K} \ell\|_{\widetilde{\mathbb{H}}_{\Theta}^{-1}(\mathcal{S}, \ell Q)}=\|\mathcal{K} \ell\|_{\mathbb{H}_{\Theta}^{-1}(\mathcal{S}, \ell Q)} .
$$

These two facts imply that the curve $(P(t))_{t_{0} \leq t<\infty}$ from Theorem 8.6, which corresponds to the original backward equation $\partial \ell_{t}=\nabla \cdot\left(\nabla \ell_{t}\right)=\mathcal{K} \ell_{t}$ of (3.9), is a curve of steepest descent also with respect to the modified $\widetilde{\mathbb{H}}_{\Theta}^{-1}(\mathcal{S}, \ell Q)$-norms in (8.23).

To prove the inequality (8.24), we use Proposition 8.3 and the identity $\mathcal{K} f=\nabla \cdot(\nabla f)$ to obtain

$$
\begin{aligned}
\|f\|_{\mathbb{H}_{\Theta}^{-1}(\mathcal{S}, \ell Q)}^{2} & =\inf _{G: \mathcal{Z} \rightarrow \mathbb{R}}\left\{\left\langle G, \vartheta_{\ell} G\right\rangle_{\mathbb{L}^{2}(\mathcal{Z}, C)}: f+\nabla \cdot\left(\vartheta_{\ell} G\right)=0\right\} \\
& \leq\left\langle\frac{1}{\vartheta_{\ell}} \nabla\left(\mathcal{K}^{-1} f\right), \vartheta_{\ell}\left(\frac{1}{\vartheta_{\ell}} \nabla\left(\mathcal{K}^{-1} f\right)\right)\right\rangle_{\mathbb{L}^{2}(\mathcal{Z}, C)}=\|f\|_{\widetilde{\mathbb{H}}_{\Theta}^{-1}(\mathcal{S}, \ell Q)}^{2} .
\end{aligned}
$$

On the one hand, the equality (8.25) holds for $f=\mathcal{K} \ell$, since

$$
\begin{aligned}
\|\mathcal{K} \ell\|_{\widetilde{\mathbb{H}}_{\Theta}^{-1}(\mathcal{S}, \ell Q)}^{2}=\left\langle\frac{1}{\vartheta_{\ell}} \nabla \ell, \nabla \ell\right\rangle_{\mathbb{L}^{2}(\mathcal{Z}, C)} & =\langle\nabla \varphi(\ell), \nabla \ell\rangle_{\mathbb{L}^{2}(\mathcal{Z}, C)} \\
& =\left\langle\nabla \varphi(\ell), \vartheta_{\ell} \nabla \varphi(\ell)\right\rangle_{\mathbb{L}^{2}(\mathcal{Z}, C)}=\|\varphi(\ell)\|_{\mathbb{H}_{\Theta}^{1}(\mathcal{S}, \ell Q)}^{2} ;
\end{aligned}
$$

while, on the other hand, Proposition 8.3 yields

$$
\|\mathcal{K} \ell\|_{\mathbb{H}_{\Theta}^{-1}(\mathcal{S}, \ell Q)}^{2}=\|\nabla \cdot(\nabla \ell)\|_{\mathbb{H}_{\Theta}^{-1}(\mathcal{S}, \ell Q)}^{2}=\left\|\nabla \cdot\left(\vartheta_{\ell} \nabla \varphi(\ell)\right)\right\|_{\mathbb{H}_{\Theta}^{-1}(\mathcal{S}, \ell Q)}^{2}=\|\varphi(\ell)\|_{\mathbb{H}_{\Theta}^{1}(\mathcal{S}, \ell Q)}^{2} .
$$

Remark 8.4. In general, the norms $\|f\|_{\widetilde{\mathbb{H}}_{\Theta}^{-1}(\mathcal{S}, \ell Q)}$ and $\|f\|_{\mathbb{H}_{\Theta}^{-1}(\mathcal{S}, \ell Q)}$ are different. Indeed, it follows from Proposition 8.3 and (8.26) that equality of norms holds if, and only if, $\frac{1}{\vartheta_{\ell}} \nabla\left(\mathcal{K}^{-1} f\right)$ is a discrete gradient. This is in general false, but is true in the following very special cases:

- At equilibrium, i.e., with $\ell \equiv 1$, we have $\vartheta_{\ell} \equiv 1$, so that $\frac{1}{\vartheta_{\ell}} \nabla\left(\mathcal{K}^{-1} f\right)=\nabla\left(\mathcal{K}^{-1} f\right)$;

- For the variance functional $\Phi(\xi)=\frac{1}{2}\left(\xi^{2}-1\right)$ as in Section $6, \vartheta_{\ell} \equiv 1$ for every likelihood ratio $\ell$;

- If the state space $\mathcal{S}$ consists of only two points, $\frac{1}{\vartheta_{\ell}} \nabla\left(\mathcal{K}^{-1} f\right)$ is a discrete gradient, since this holds for every anti-symmetric function on $\mathcal{S} \times \mathcal{S}$. 


\section{Gradient Flows}

Let us reconsider now, under conditions of detailed balance, the results of Sections 6-9 from a different, "Riemannian" point of view. We shall see here that, under the conditions (3.11), the curve $(P(t))_{0 \leq t<\infty}$ of time-marginal distributions for the Chain evolves as a gradient flow of the relative $\Phi-$ entropy. This takes place in a suitable geometry on the space of probability measures, in the spirit of the pioneering work by Jordan, Kinderlehrer \& OtTO (1998). We refer to ERBAR \& MAAS (2012, 2014), Mielke (2011, 2013) and to the expository paper MAAS (2017), for an in-depth study of such issues in discrete spaces.

We summon from subsection 3.1 the manifold $\mathcal{M}=\mathcal{P}_{+}(\mathcal{S})$ of probability vectors $P=(p(x))_{x \in \mathcal{S}}$ with strictly positive entries; i.e., $\mathcal{M}$ is the interior of the lateral face of the unit simplex in $\mathbb{R}^{n}$, with $n=|\mathcal{S}|$ the cardinality of the state-space. We denote by $\mathcal{M}_{0}(\mathcal{S})$ the collection of vectors $W=(w(x))_{x \in \mathcal{S}}$ with total mass $\sum_{x \in \mathcal{S}} w(x)=0$, viewed as "signed measures", and observe that $\mathcal{M}$ is a relatively open subset of the $(n-1)$-dimensional affine space $P+\mathcal{M}_{0}(\mathcal{S})=\left\{P+W: W \in \mathcal{M}_{0}(\mathcal{S})\right\}$, for an arbitrary $P \in \mathcal{M}$. This observation allows us to identify the tangent space at each $P \in \mathcal{M}$ with $\mathcal{M}_{0}(\mathcal{S})$.

\subsection{Gradient Flow for the Variance}

As a warmup, let us start as in Section 6 with a derivation for the gradient flow property for the variance functional $\mathcal{M} \ni P \mapsto V(P \mid Q) \in \mathbb{R}_{+}$of (6.1). Following DE GIORGI's approach to curves of maximal slope (cf. Ambrosio, Gigli \& SAVARÉ (2008)), we compute the dissipation of this functional along an arbitrary smooth curve $\left(\widetilde{P}_{t}\right)_{0 \leq t<\infty}$ on $\mathcal{M}$; or equivalently, along the curve $\left(\widetilde{\ell}_{t}\right)_{0 \leq t<\infty}$ induced on the space $\mathcal{L}$ by the likelihood ratios $\widetilde{\ell}_{t}(y)=\widetilde{p}_{t}(y) / q(y), y \in \mathcal{S}$.

As in Section 6, we express the time-evolution of this likelihood ratio curve as $\partial \widetilde{\ell}_{t}=\mathcal{K} f_{t}=\nabla \cdot\left(\nabla f_{t}\right)$ in the manner of (3.9), for a suitable curve $\left(f_{t}\right)_{0 \leq t<\infty}$ of functions $f_{t}: \mathcal{S} \rightarrow \mathbb{R}$. This is uniquely determined up to an additive constant on account of the Chain's irreducibility, and its discrete gradient provides the "momentum vector field" of the motion. Recalling the consequences $\widehat{\mathcal{K}} f=\mathcal{K} f=\nabla \cdot(\nabla f)$ of detailed balance (3.11) and of (4.3), as well as the fact that $\nabla \cdot$ is the adjoint of $-\nabla$ from (4.10), we obtain

$$
\begin{aligned}
\partial V\left(\widetilde{P}_{t} \mid Q\right) & =\partial\left\|\widetilde{\ell}_{t}\right\|_{\mathbb{L}^{2}(\mathcal{S}, Q)}^{2}=2\left\langle\widetilde{\ell}_{t}, \partial \widetilde{\ell}_{t}\right\rangle_{\mathbb{L}^{2}(\mathcal{S}, Q)}=2\left\langle\widetilde{\ell}_{t}, \mathcal{K} f_{t}\right\rangle_{\mathbb{L}^{2}(\mathcal{S}, Q)}=-2\left\langle\nabla \widetilde{\ell}_{t}, \nabla f_{t}\right\rangle_{\mathbb{L}^{2}(\mathcal{Z}, C)} \\
& \geq-2\left\|\nabla \widetilde{\ell}_{t}\right\|_{\mathbb{L}^{2}(\mathcal{Z}, C)}\left\|\nabla f_{t}\right\|_{\mathbb{L}^{2}(\mathcal{Z}, C)} \geq-\left\|\nabla \widetilde{\ell}_{t}\right\|_{\mathbb{L}^{2}(\mathcal{Z}, C)}^{2}-\left\|\nabla f_{t}\right\|_{\mathbb{L}^{2}(\mathcal{Z}, C)}^{2} .
\end{aligned}
$$

Equality holds in the first (resp., the second) of these inequalities if, and only if, $\nabla f_{t}$ and $\nabla \widetilde{\ell}_{t}$ are positively collinear (resp., have the same norm). In other words, both these last two inequalities hold as equalities if and only if $\nabla f_{t}=\nabla \widetilde{\ell}_{t}$, and this leads to the backwards equation (3.9) on account of detailed balance:

$$
\partial \widetilde{\ell}_{t}=\mathcal{K} f_{t}=\nabla \cdot\left(\nabla f_{t}\right)=\nabla \cdot\left(\nabla \tilde{\ell}_{t}\right)=\mathcal{K} \tilde{\ell}_{t}=\widehat{\mathcal{K}} \tilde{\ell}_{t} .
$$

But the last two norms in (9.1) are then $\left\|\nabla f_{t}\right\|_{\mathbb{L}^{2}(\mathcal{Z}, C)}=\left\|\nabla\left(\mathcal{K}^{-1}\left(\partial \widetilde{\ell}_{t}\right)\right)\right\|_{\mathbb{L}^{2}(\mathcal{Z}, C)}=\left\|\partial \widetilde{\ell}_{t}\right\|_{\mathbb{H}^{-1}(\mathcal{S}, Q)}$ as well as $\left\|\nabla \widetilde{\ell}_{t}\right\|_{\mathbb{L}^{2}(\mathcal{Z}, C)}=\left\|\widetilde{\ell}_{t}\right\|_{\mathbb{H}^{1}(\mathcal{S}, Q)}$.

In this manner we obtain from (9.1) the following classical result. This provides another proof for Theorem 6.3 by identifying the solutions of $\partial P_{t}=\mathcal{K}^{\prime} P_{t}$ in (3.5) as curves in the direction of steepest descent for the variance, relative to the distance induced by the $\mathbb{H}^{-1}(\mathcal{S}, Q)$ norm. But it also strengthens Theorem 6.3, by identifying also the correct velocity with which the gradient flow moves into this direction.

Theorem 9.1. For any given probability vector $P \in \mathcal{M}$ and with $\ell \in \mathcal{L}$ the likelihood ratio vector corresponding to $P$, we have along any smooth curve $\left(\widetilde{P}_{t}\right)_{0 \leq t<\infty}$ on $\mathcal{M}$ with $\widetilde{P}_{0}=P$ the inequality

$$
\left.\left(\partial V\left(\widetilde{P}_{t} \mid Q\right)+\left\|\partial \widetilde{\ell}_{t}\right\|_{\mathbb{H}^{-1}(\mathcal{S}, Q)}^{2}\right)\right|_{t=0} \geq-\|\ell\|_{\mathbb{H}^{1}(\mathcal{S}, Q)}^{2} .
$$


Equality holds if, and only if, the curve $\left(\widetilde{P}_{t}\right)_{0 \leq t<\infty} \subset \mathcal{M}$ satisfies the forward equation $\partial \widetilde{P}_{t}=\mathcal{K}^{\prime} \widetilde{P}_{t}$ (equivalently, the induced likelihood ratio curve $\left(\widetilde{\ell}_{t}\right)_{0 \leq t<\infty} \subset \mathcal{L}$ satisfies the backward equation $\left.\partial \widetilde{\ell}_{t}=\mathcal{K} \widetilde{\ell}_{t}\right)$.

\subsection{Gradient Flow for the $\Phi-$ Relative Entropy}

Let us examine now, how these ideas might work in the context of the generalized relative entropy functional

$$
\mathcal{M} \ni P \longmapsto H^{\Phi}(P \mid Q):=\sum_{y \in \mathcal{S}} q(y) \Phi\left(\frac{p(y)}{q(y)}\right) \in[0, \infty)
$$

corresponding to a convex function $\Phi$, as in Section 8. We fix a smooth curve $\left(\widetilde{P}_{t}\right)_{0 \leq t<\infty}$ on $\mathcal{M}$ emanating from a given $\widetilde{P}_{0}=P \in \mathcal{M}$; and consider the induced curve $\left(\widetilde{\ell}_{t}\right)_{0 \leq t<\infty} \subset \mathcal{L}$ of likelihood ratios $\widetilde{\ell}_{t}(y)=$ $\widetilde{p}_{t}(y) / q(y), y \in \mathcal{S}$ emanating from $\boldsymbol{\ell}=\ell_{0}$.

As in subsection 8.3, we cast the time-evolution of the likelihood ratio curve as a continuity equation

$$
\partial \widetilde{\ell}_{t}+\nabla \cdot\left(\widetilde{\vartheta}_{t} \nabla f_{t}\right)=0
$$

where the "velocity vector field" is the discrete gradient of a suitable function $f_{t}: \mathcal{S} \rightarrow \mathbb{R}$, and $\widetilde{\vartheta}_{t}$ is a shorthand for $\vartheta_{\widetilde{\ell_{t}}}$ from (8.13). In the manner of (9.1), this expresses the time-evolution of the $\Phi$-relative entropy functional $H^{\Phi}\left(\widetilde{P}_{t} \mid Q\right)=\sum_{y \in \mathcal{S}} q(y) \Phi\left(\widetilde{\ell}_{t}(y)\right)$ in (9.2) along the curve $\left(\widetilde{P}_{t}\right)_{0 \leq t<\infty}$ as

$$
\begin{aligned}
\partial H^{\Phi}\left(\widetilde{P}_{t} \mid Q\right) & =\left\langle\varphi\left(\widetilde{\ell}_{t}\right), \partial \widetilde{\ell}_{t}\right\rangle_{\mathbb{L}^{2}(\mathcal{S}, Q)}=-\left\langle\varphi\left(\widetilde{\ell}_{t}\right), \nabla \cdot\left(\widetilde{\vartheta}_{t} \nabla f_{t}\right)\right\rangle_{\mathbb{L}^{2}(\mathcal{S}, Q)}=\left\langle\nabla \varphi\left(\widetilde{\ell}_{t}\right), \widetilde{\vartheta}_{t} \nabla f_{t}\right\rangle_{\mathbb{L}^{2}(\mathcal{Z}, C)} \\
& =\left\langle\nabla \varphi\left(\widetilde{\ell}_{t}\right), \nabla f_{t}\right\rangle_{\mathbb{L}^{2}\left(\mathcal{Z}, \widetilde{\vartheta}_{t} C\right)} \geq-\left\|\nabla \varphi\left(\widetilde{\ell}_{t}\right)\right\|_{\mathbb{L}^{2}\left(\mathcal{Z}, \widetilde{\vartheta}_{t} C\right)}\left\|\nabla f_{t}\right\|_{\mathbb{L}^{2}\left(\mathcal{Z}, \widetilde{\vartheta}_{t} C\right)} \\
& \geq-\left(\left\|\nabla \varphi\left(\widetilde{\ell}_{t}\right)\right\|_{\mathbb{L}^{2}\left(\mathcal{Z}, \widetilde{\vartheta}_{t} C\right)}^{2}+\left\|\nabla f_{t}\right\|_{\mathbb{L}^{2}\left(\mathcal{Z}, \widetilde{\vartheta}_{t} C\right)}^{2}\right) / 2 .
\end{aligned}
$$

Once again, equality holds if and only if $\nabla f_{t}=\nabla\left(\varphi\left(\widetilde{\ell}_{t}\right)\right)$, and this leads by detailed balance to the backwards equation

$$
\partial \tilde{\ell}_{t}=-\nabla \cdot\left(\widetilde{\vartheta}_{t} \nabla f_{t}\right)=\nabla \cdot\left(\tilde{\vartheta}_{t} \nabla\left(\varphi\left(\tilde{\ell}_{t}\right)\right)\right)=\nabla \cdot\left(\nabla \tilde{\ell}_{t}\right)=\mathcal{K} \tilde{\ell}_{t}=\widehat{\mathcal{K}} \tilde{\ell}_{t}
$$

of (3.9). We have used here the elementary but crucial consequence $\widetilde{\vartheta}_{t} \nabla\left(\varphi\left(\widetilde{\ell}_{t}\right)\right)=\nabla \tilde{\ell}_{t}$ of $(8.13)$, a "discrete chain rule" that sheds light on our choice of weight-function $\Theta^{\Phi}$ in (8.12). But the last two norms displayed in (9.4) are $\left\|\nabla f_{t}\right\|_{\mathbb{L}^{2}\left(\mathcal{Z}, \widetilde{\vartheta}_{t} C\right)}=\left\|\partial \widetilde{\ell}_{t}\right\|_{\mathbb{H}_{\Theta}^{-1}\left(\mathcal{S}, \widetilde{\ell}_{t} Q\right)}$ and $\left\|\nabla \varphi\left(\widetilde{\ell}_{t}\right)\right\|_{\mathbb{L}^{2}\left(\mathcal{Z}, \widetilde{\vartheta}_{t} C\right)}=\left\|\varphi\left(\widetilde{\ell}_{t}\right)\right\|_{\mathbb{H}_{\Theta}^{1}\left(\mathcal{S}, \widetilde{\ell}_{t} Q\right)}$.

We summarize the situation in Theorem 9.2 below; this corresponds to Theorem 8.6 , in the same manner as Theorem 9.1 corresponds to Theorem 6.3. Again, the DE GIORGI argument (9.4) gives not only the "direction of steepest descent" into which the gradient flow travels, but also the velocity of this flow.

Theorem 9.2. For any given probability vector $P \in \mathcal{M}$, and with $\ell \in \mathcal{L}$ the likelihood ratio vector corresponding to $P$, we have along any smooth curve $\left(\widetilde{P}_{t}\right)_{0 \leq t<\infty}$ on $\mathcal{M}$ with $\widetilde{P}_{0}=P$ the inequality

$$
\left.\left(2 \partial H^{\Phi}\left(\widetilde{P}_{t} \mid Q\right)+\left\|\partial \widetilde{\ell}_{t}\right\|_{\mathbb{H}_{\Theta}^{-1}(\mathcal{S}, \ell Q)}^{2}\right)\right|_{t=0} \geq-\|\varphi(\ell)\|_{\mathbb{H}_{\Theta}^{1}(\mathcal{S}, \ell Q)}^{2} .
$$

Equality holds here if, and only if, the curve $\left(\widetilde{P}_{t}\right)_{0 \leq t<\infty} \subset \mathcal{M}$ satisfies the forward equation $\partial \widetilde{P}_{t}=\mathcal{K}^{\prime} \widetilde{P}_{t}$ (equivalently, the likelihood ratio curve $\left(\widetilde{\ell}_{t}\right)_{0 \leq t<\infty} \subset \mathcal{L}$ satisfies the backward equation $\partial \widetilde{\ell}_{t}=\mathcal{K} \widetilde{\ell}_{t}$, and the corresponding "driver" in (9.3) is $f_{t}=-\varphi\left(\widetilde{\ell}_{t}\right)$.) 


\subsection{A Riemannian Framework}

Let us take up these same ideas again, but now in a Riemannian-geometric framework as for instance in MAAS (2011), Mielke (2011). For any given probability vector $P \in \mathcal{M}$, we define the "likelihood ratio" vector $\ell=(\ell(x))_{x \in \mathcal{S}} \in \mathcal{L}$ with strictly positive elements $\ell(x):=p(x) / q(x)$. We consider then the Riemannian metric $\left(g_{\ell}\right)_{\ell \in \mathcal{L}}$ on $\mathcal{L}$ induced by the scalar products

$$
g_{\ell}\left(\partial \ell_{1}, \partial \ell_{2}\right):=\left\langle\nabla \psi_{1}, \nabla \psi_{2}\right\rangle_{\mathbb{L}^{2}\left(\mathcal{Z}, \vartheta_{\ell} C\right)},
$$

where $\nabla \psi_{i}$ is the unique discrete gradient satisfying the equation of "continuity type" $\partial \ell_{i}=\nabla \cdot\left(\vartheta_{\ell} \nabla \psi_{i}\right)$ for $i=1,2$. In particular, $g_{\ell}(\partial \ell, \partial \ell)=\|\nabla \psi\|_{\mathbb{L}^{2}\left(\mathcal{Z}, \vartheta_{\ell} C\right)}^{2}=\|\partial \ell\|_{\mathbb{H}_{\Theta}^{-1}(\mathcal{S}, \ell Q)}^{2}$ on account of (8.17).

The Riemannian gradient grad $F$ of a smooth functional $F: \mathcal{L} \rightarrow \mathbb{R}$ is then given by

$$
\operatorname{grad} F=-\nabla \cdot\left(\vartheta_{\ell} \nabla D_{\ell} F\right), \quad \text { where } \quad D_{\ell} F \equiv \frac{\delta F}{\delta \ell}
$$

is the $\mathbb{L}^{2}(\mathcal{S}, Q)$-derivative defined by $\lim _{\varepsilon \rightarrow 0} \varepsilon^{-1}(F(\ell+\varepsilon \eta)-F(\ell))=\left\langle D_{\ell} F, \eta\right\rangle_{\mathbb{L}^{2}(\mathcal{S}, Q)}$ for $\eta: \mathcal{S} \rightarrow \mathbb{R}$ with $\sum_{x \in \mathcal{X}} \eta(x) q(x)=0$. In particular, the gradient flow equation $\partial \ell=-\operatorname{grad} F(\ell)$ reads

$$
\partial \ell=\nabla \cdot\left(\vartheta_{\ell} \nabla D_{\ell} F\right) .
$$

The Riemannian metric $g$ on $\mathcal{L}$ can be turned into a Riemannian metric $G$ on the manifold of probability measures $\mathcal{M}$, via $G_{P}\left(\partial P_{1}, \partial P_{2}\right):=g_{\ell}\left(\partial \ell_{1}, \partial \ell_{2}\right)$, where $P=\ell Q$ and $\partial P_{i}=\partial \ell_{i} Q$ for $i=1,2$.

Theorem 9.3. (MAAS (2011), MIELKE (2011)): Under the detailed balance conditions (3.11), and with $\Theta$ the function of (8.12), the Forward Kolmogorov equation $\partial P(t)=\mathcal{K}^{\prime} P(t)$ in (3.5) is the gradient flow of the $\Phi$-relative entropy in (9.2) with respect to the Riemannian metric $G$ induced on the manifold $\mathcal{M}$.

Proof: Let $(P(t))_{0 \leq t<\infty}$ solve the Forward Kolmogorov equation $\partial P(t)=\mathcal{K}^{\prime} P(t)$. By detailed balance, the associated likelihood ratio curve $(\ell(t))_{0 \leq t<\infty} \subset \mathcal{L}$ satisfies the backward equation $\partial \boldsymbol{\ell}(t)=$ $\mathcal{K} \ell(t)$. In view of (9.6), we thus need to verify the identity

$$
\mathcal{K} \ell=\nabla \cdot\left(\vartheta_{\ell} \nabla D_{\ell} h^{\Phi}\right)
$$

where $h^{\Phi}: \mathcal{L} \rightarrow \mathbb{R}$ is defined by $h^{\Phi}(\ell)=H^{\Phi}(\ell Q \mid Q)$.

For $\ell \in \mathcal{L}$ and $\eta: \mathcal{S} \rightarrow \mathbb{R}$ with $\sum_{x \in \mathcal{S}} \eta(x) q(x)=0$, we have the directional derivative computation

$$
\left.\frac{\mathrm{d}}{\mathrm{d} \varepsilon} h^{\Phi}(\ell+\varepsilon \eta)\right|_{\varepsilon=0}=\sum_{x \in \mathcal{S}} \eta(x) \varphi(\ell(x)) ; \quad \text { thus } \quad D_{\ell} h^{\Phi} \equiv \frac{\delta h^{\Phi}}{\delta \ell}=\varphi(\ell):=(\varphi(\ell(x)))_{x \in \mathcal{S}}
$$

Invoking the "discrete chain-rule" $\vartheta_{\ell} \nabla(\varphi(\ell))=\nabla \ell$ we obtain the desired identity

$$
\nabla \cdot\left(\vartheta_{\ell} \nabla D_{\ell} h^{\Phi}\right)=\nabla \cdot\left(\vartheta_{\ell} \nabla(\varphi(\ell))\right)=\nabla \cdot(\nabla \ell)=\mathcal{K} \ell
$$

Theorem 9.3 has a converse, developed in DIETERT (2015) as follows.

Proposition 9.4. Suppose that there exists a $\mathcal{C}^{1}$ Riemannian metric on the manifold of probability vectors $\mathcal{M}$, under which the Forward Kolmogorov equation $\partial P(t)=\mathcal{K}^{\prime} P(t)$ of $(3.5)$ is the gradient flow for the relative entropy in (7.1). Then the MARKOV Chain satisfies the detailed balance conditions (3.11). 


\subsection{The HWI Inequality}

In the Riemannian framework of this Section, we present now a version of the celebrated HWI inequality of OTTO \& VILLANI (2000). The basic ingredient is the notion of RICCI curvature in the present context, as in Definition 1.3 of MAAS (2011). We recast this definition using the more general notion of $\Phi$-entropy in Section 8 - rather than the classical entropy which is, of course, a special case. We recall also from subsection 3.1, Remark 5.1 the manifold $\mathcal{M}$ of probability vectors on $\mathcal{S}$ with strictly positive entries, its closure $\overline{\mathcal{M}}$ of probability vectors with nonnegative entries, and the corresponding manifolds $\mathcal{L}, \overline{\mathcal{L}}$ of likelihood ratios.

Definition 9.1. Ricci $^{\Phi}$-curvature: We say that our finite-state MARKOV Chain with generator $\mathcal{K}$ has nonlocal RICCI curvature bounded from below by $\kappa \in \mathbb{R}$ relative to $\Phi$ as above, and write $\operatorname{Ricci}^{\Phi}(\mathcal{K}) \geq \kappa$, if for every constant-speed geodesic $\left(P_{t}\right)_{0 \leq t \leq 1}$ on the closed manifold $\overline{\mathcal{M}}$ we have the inequality

$$
H^{\Phi}\left(P_{t} \mid Q\right) \leq(1-t) H^{\Phi}\left(P_{0} \mid Q\right)+t H^{\Phi}\left(P_{1} \mid Q\right)-\frac{\kappa}{2} t(1-t) \mathcal{W}^{2}\left(P_{0}, P_{1}\right), \quad 0 \leq t \leq 1 .
$$

Here $\mathcal{W}(\cdot, \cdot)$ is the geodesic distance with respect to the Riemannian metric of subsection 9.3. It admits the BENAMOU-BRENIER-type representation

$$
\mathcal{W}^{2}\left(P_{0}, P_{1}\right)=\inf \left\{\int_{0}^{1}\left\|f_{t}\right\|_{\mathbb{H}_{\Theta}^{1}\left(\mathcal{S}, \tilde{\ell}_{t} Q\right)}^{2} \mathrm{~d} t: \partial \widetilde{\ell}_{t}+\nabla \cdot\left(\widetilde{\vartheta}_{t} \nabla f_{t}\right)=0\right\},
$$

with the infimum running over all solutions to the continuity equation connecting $P_{0} \equiv \widetilde{\ell}_{0} Q$ with $P_{1} \equiv$ $\widetilde{\ell}_{1} Q$; cf. MAAS (2011), ERbAR \& MAAS (2012), Mielke (2013). We shall apply the above inequality (9.8) in the form of the following fact about functions of a real variable.

Proposition 9.5. Let $(f(t))_{0 \leq t \leq 1}$ be a continuous, real-valued function such that

$$
f(t+h)-2 f(t)+f(t-h) \geq \kappa h^{2}
$$

holds for some $\kappa \in \mathbb{R}$ and every pair $(t, h) \in \mathbb{R}_{+}^{2}$ with $h \leq t \leq 1-h$. Suppose also that $f$ is rightdifferentiable at $t=0$ with derivative $f^{\prime}(0)$. Then

$$
f(1) \geq f(0)+f^{\prime}(0)+\frac{\kappa}{2} .
$$

Proof: If $f$ is twice differentiable, the condition (9.10) amounts to $f^{\prime \prime} \geq \kappa$. For general $f$ and supposing $\kappa=0$, condition (9.10) is tantamount to the convexity of $f$, so the inequality (9.11) becomes obvious. The case of general $\kappa$ follows by subtracting from $f(t)$ the quadratic $\kappa t^{2} / 2$.

For a constant-speed geodesic $\left(P_{t}\right)_{0 \leq t \leq 1}$ joining $P_{0} \in \mathcal{M}$ with $P_{1} \in \overline{\mathcal{M}}$ such that $\mathcal{W}\left(P_{0}, P_{1}\right)=1$, the function $f(t)=H^{\Phi}\left(P_{t} \mid Q\right)$ satisfies the conditions of Proposition 9.5 under the assumption $\operatorname{Ricci}^{\Phi}(\mathcal{K}) \geq$ $\kappa$. Indeed, $\left(P_{u}\right)_{t-h \leq u \leq t+h}$ is then a constant-speed geodesic which joins $P_{t-h}$ with $P_{t+h}$ and satisfies $\mathcal{W}\left(P_{t-h}, P_{t+h}\right)=2 h$, so (9.8) applies with $t=1 / 2$. The existence of constant-speed geodesics and of $f^{\prime}(0)$, follows respectively from Theorem 3.2 and Proposition 3.4 in ERBAR \& MAAS (2012).

We formulate now a version of the HWI inequality in the present context. This sharpens slightly Theorem 7.3 of ERBAR \& MAAS (2012), where $P_{1}$ in the following theorem is the invariant measure $Q$; and its proof does not rely on the "evolution variational inequality" (the EVI of Theorem 4.5 in ERBAR \& MAAS (2012)), but rather on the very elementary estimate of Proposition 9.5.

Theorem 9.6. HWI Inequality of OTTO-VILLANI: Under the assumptions of subsection 9.2, suppose that $\operatorname{Ricci}^{\Phi}(\mathcal{K}) \geq \kappa$ holds for some $\kappa \in \mathbb{R}$. With $P_{0}, P_{1}$ any probability measures in $\mathcal{M}, \overline{\mathcal{M}}$, respectively, denote by $\mathcal{W}\left(P_{0}, P_{1}\right)$ their geodesic distance and by $I^{\Phi}\left(P_{0} \mid Q\right)$ the $\Phi$-FISHER information of (8.8) with $t=0$. We have then

$$
H^{\Phi}\left(P_{0} \mid Q\right)-H^{\Phi}\left(P_{1} \mid Q\right) \leq\left(I^{\Phi}\left(P_{0} \mid Q\right)\right)^{1 / 2} \mathcal{W}\left(P_{0}, P_{1}\right)-\frac{\kappa}{2} \mathcal{W}^{2}\left(P_{0}, P_{1}\right)
$$


Proof: We follow the argument in Theorem 4.11 of KARATZAS, SCHACHERMAYER \& TSCHIDERER (2020), where the HWI inequality is established for diffusions in $\mathbb{R}^{n}$. We let $\left(P_{t}\right)_{0 \leq t \leq 1} \subset \overline{\mathcal{M}}$ be a constantspeed geodesic of probability measures joining $P_{0}$ with $P_{1}$ (which we know exists, by Theorem 3.2 of ERBAR \& MAAS (2012)), denote by $\left(\ell_{t}\right)_{0 \leq t \leq 1} \subset \overline{\mathcal{L}}$ the corresponding likelihood-ratio curve, consider the function $f(t):=H^{\Phi}\left(P_{t} \mid Q\right), 0 \leq t \leq 1$, and pass to the parametrization

$$
u=u(t)=\frac{w}{i^{1 / 2}} t, \quad 0 \leq u \leq \frac{w}{i^{1 / 2}},
$$

where $i=I^{\Phi}\left(P_{0}\right)=\mathcal{E}\left(\ell_{0}, \phi\left(\ell_{0}\right)\right)$ and $w=\mathcal{W}\left(P_{0}, P_{1}\right)$. We set $g(u)=g(u(t))=f(t)$. Recalling the likelihood ratio $\ell_{t}$ corresponding to $P_{t}$, consider the continuous curve of likelihood ratios

$$
\tilde{\ell}(u)=\ell_{t}, \quad 0 \leq u \leq \frac{w}{i^{1 / 2}}
$$

so that $\widetilde{\ell}(0)=\ell_{0}$ and $\widetilde{\ell}\left(w i^{-1 / 2}\right)=\ell_{1}$, as well as the corresponding curve $\widetilde{P}(u), 0 \leq u \leq w i^{-1 / 2}$ of probabilities. Since $\left(P_{t}\right)_{0 \leq t \leq 1}$ is a geodesic of constant speed $w$ with $\ell=\ell_{0}$, we have

$$
\left\|\partial \ell_{0}\right\|_{H_{\Theta}^{-1}(\mathcal{S}, \ell Q)}=\mathcal{W}\left(P_{0}, P_{1}\right)=w, \quad \text { thus } \quad\|\partial \widetilde{\ell}(0)\|_{H_{\Theta}^{-1}(\mathcal{S}, \ell Q)}^{2}=i
$$

this last display gives the second term in (9.5). As for the term $\|\varphi(\ell)\|_{\mathbb{H}_{\Theta}^{1}(\mathcal{S}, \ell Q)}^{2}$ in (9.5), the expression (8.8) and Remark 8.3 give $\|\varphi(\widetilde{\ell}(0))\|_{H_{\Theta}^{1}(\mathcal{S}, \ell Q)}^{2}=\mathcal{E}\left(\ell_{0}, \varphi\left(\ell_{0}\right)\right)=i$. In this manner, (9.5) leads to the inequality

$$
g^{\prime}(0)=\left.\partial H^{\Phi}\left(\widetilde{P}_{u} \mid Q\right)\right|_{u=0} \geq-i,
$$

where the existence of the right-derivative $g^{\prime}(0)$ is assured by Proposition 3.4 of ERBAR \& MAAS (2012).

Going back to the original parametrization, we obtain $f^{\prime}(0) \geq-w i^{1 / 2}$. The assumption $\operatorname{Ricci}^{\Phi}(\mathcal{K}) \geq \kappa$ implies that $f$ satisfies (9.10), with $\kappa$ replaced by $\kappa w^{2}$. In conclusion, (9.11) gives

$$
H^{\Phi}\left(P_{1} \mid Q\right) \geq H^{\Phi}\left(P_{0} \mid Q\right)-i^{1 / 2} w+\frac{\kappa}{2} w^{2},
$$

which is tantamount to the HWI inequality (9.12).

Remark 9.1. As is well known (e.g., ERBAR \& MAAS (2012)), the HWI inequality leads directly to the corresponding versions of the Modified Log-SOBOLEV and TALAGRAND inequalities, by taking $\Phi(\cdot)=$ $\Psi(\cdot)$ as in (7.13) and $P_{1}=Q$. POINCARÉ-type inequalities also follow this way, by linearizing the Modified Log-SOBOLEV inequality.

The HWI inequality (9.12) can be sharpened. In the above proof, we estimated the slope of the function $t \mapsto H^{\Phi}\left(P_{t} \mid Q\right)$ at $t=0$ in terms of the "worst case", i.e., the steepest possible descent; this led to the square root of the FISHER information, by Theorem 8.6. But Propositions 8.4, 8.5 allow us to calculate the slope of this function with respect to the norm $H_{\Theta}^{-1}(\mathcal{S}, \ell \mathcal{Q})$, which induces the local Riemannian metric at $\ell=\ell_{0}$. We obtain in this manner the following more precise result, in the spirit of OTTO \& VILLANI (2000), Cordero-Erausquin (2002) or Karatzas, Schachermayer \& Tschiderer (2020).

Proposition 9.7. Under the assumptions of Theorem 9.6, suppose in addition that the curve $\left(P_{t}\right)_{0 \leq t \leq 1}$ is driven by a continuous function $\left(\psi_{t}\right)_{0 \leq t \leq 1}$ via the "discrete continuity equation"

$$
\partial \ell_{t}^{\psi}+\nabla \cdot\left(\vartheta_{\ell_{t}} \nabla \psi_{t}\right)=0 .
$$

Then with $\ell=\ell_{0}$, we have the inequality

$$
H^{\Phi}\left(P_{0} \mid Q\right)-H^{\Phi}\left(P_{1} \mid Q\right) \leq \mathcal{W}\left(P_{0}, P_{1}\right)\left\langle\varphi(\ell), \frac{\psi_{0}}{\left\|\psi_{0}\right\|_{\mathbb{H}_{\Theta}^{1}(\mathcal{S}, \ell Q)}}\right\rangle_{\mathbb{H}_{\Theta}^{1}(\mathcal{S}, \ell Q)}-\frac{\kappa}{2} \mathcal{W}^{2}\left(P_{0}, P_{1}\right) .
$$


Proof. From (8.22), the slope of the function $H^{\Phi}\left(P_{t} \mid Q\right)$ with respect to the norm $H_{\Theta}^{-1}(\mathcal{S}, \ell \mathcal{Q})$ on $\mathcal{M}$, which induces the local Riemannian metric at $(t, \ell)=\left(0, \ell_{0}\right)$, is given by the bracket term on the right hand side of (9.15). Hence, we may replace the inequality (9.13) by the more precise equality

$$
g^{\prime}(0)=-\left\langle\varphi\left(\ell_{0}\right), \frac{\psi_{0}}{\left\|\psi_{0}\right\|_{\mathbb{H}_{\Theta}^{1}(\mathcal{S}, \ell Q)}}\right\rangle_{\mathbb{H}_{\Theta}^{1}(\mathcal{S}, \ell Q)} .
$$

The rest of the proof of Theorem 9.6 can be repeated verbatim, to arrive as (9.15) instead of (9.12).

Remark 9.2. What happens when $P_{0}$ is on the boundary of $\mathcal{M}$, as in Remark 5.1? That is, when the set $\mathcal{N}_{0}=\left\{x \in \mathcal{S}: P_{0}(x)=0\right\}$ is non-empty? To be specific, let us concentrate on the classical entropy $\Phi(\ell)=\ell \log \ell$. Then the FISHER information $I^{\Phi}\left(P_{0} \mid Q\right)$ is infinite, and the HWI inequality (9.12) holds trivially. On the other hand, the refined version (9.15) may deliver some nontrivial information.

Indeed, suppose that $\left(P_{t}\right)_{0 \leq t \leq 1}$ is driven by a continuous function $\left(\psi_{t}\right)_{0 \leq t \leq 1}$ via the "discrete continuity equation" (9.14). If $\psi_{0}$ also vanishes on $\mathcal{N}_{0}$, the bracket term in (9.15) is finite (via the rule $0 \cdot \infty=0$ ). As we assume that $\psi(\cdot)$ is continuous (actually, we only need this continuity at $t=0$ ), we can still apply the above argument and conclude that (9.15) holds, yielding a nontrivial result. The geometric interpretation of $\psi_{0}$ vanishing on $\mathcal{N}_{0}$, is that the curve $\left(P_{t}\right)_{0 \leq t \leq 1}$ starts "tangentially to the boundary of $\mathcal{M}$ ", when departing from $P_{0}$ at this boundary.

\section{Countable State-Space}

It is well known that the results of Sections 2 and 3 hold also for countably infinite state-spaces $\mathcal{S}$; see Chapters 2, 3 in NoRRIS (1997) and LIGGETT (2010). In particular, the ergodic property (3.7) holds at least for bounded functions $f: \mathcal{S} \rightarrow \mathbb{R}$. The crucial Proposition 5.2 also remains valid.

Propositions 6.1, 6.2 carry over to countable state-spaces under the assumption $V(P(0) \mid Q)<\infty$. To see this, we start by observing that we can guarantee now prima facie only the local martingale property of the processes $\widehat{M}$ in (6.2). Still, we can localize $\widehat{M}$ by an increasing sequence $\left\{\sigma_{n}\right\}_{n \in \mathbb{N}}$ of $\widehat{G}-$ stoppingtimes with values in $[0, T]$ and $\lim _{n \rightarrow \infty} \uparrow \sigma_{n}=T$, and create the bounded $(\widehat{\mathbb{G}}, \mathbb{Q})$-martingales $\widehat{M}(s \wedge$ $\left.\sigma_{n}\right), 0 \leq s \leq T$. Taking expectations in (6.2) with $s=\sigma_{n}$, then letting $n \rightarrow \infty$ and using monotone convergence, the $\mathbb{Q}$-submartingale property of $\ell^{2}(T-s, \widehat{X}(s)), \quad 0 \leq s \leq T$ from Proposition 5.2, and optional sampling, we obtain from (4.9) the inequality

$$
\mathbb{E}^{\mathbb{Q}}\left[\ell^{2}(T, X(T))\right]+\int_{0}^{T} 2 \mathcal{E}\left(\ell_{t}, \ell_{t}\right) \mathrm{d} t=\lim _{n \rightarrow \infty} \uparrow \mathbb{E}^{\mathbb{Q}}\left[\ell^{2}\left(T-\sigma_{n}, \widehat{X}\left(\sigma_{n}\right)\right)\right] \leq \mathbb{E}^{\mathbb{Q}}\left[\ell^{2}(0, X(0))\right] .
$$

But the reverse of this last inequality also holds, on account of FATOU's Lemma; thus (6.7) follows for countable state-spaces as well, and $\widehat{M}$ is seen to be a true $(\widehat{\mathbb{G}}, \mathbb{Q})$-martingale. Then $\lim _{t \rightarrow \infty} V(P(t) \mid Q)=0$, and with it (6.8), are proved for a countable state-space in the manner of Proposition 10.1 below.

\subsection{Relative Entropy Dissipates all the way down to Zero}

When the state-spaces $\mathcal{S}$ is countably infinite, the results of Section 7 pertaining to the relative entropy need the additional assumption

$$
H(P(0) \mid Q)=\sum_{y \in \mathcal{S}} p(0, y) \log \left(\frac{p(0, y)}{q(y)}\right)<\infty .
$$

Then everything goes through as before, including the non-negativity and decrease claims in (7.3) - except for the argument establishing (7.9), which uses the finiteness of the state-space in a crucial manner.

Here is a proof for this result in the countable case. 
Proposition 10.1. The dissipation of relative entropy all the way down to zero, as in (7.9), holds for a countable state-space under the condition (10.1).

Proof: Let us recall the likelihood ratio process $L(t):=\ell(t, X(t)), 0 \leq t<\infty$ of (5.4), and from (5.8) that its time-reversal $L(T-s), 0 \leq s \leq T$ is a $(\widehat{\mathbb{G}}, \mathbb{Q})$-martingale.

Fix $0 \leq t_{1}<t_{2}<\infty$. For any $T \in\left(t_{2}, \infty\right)$, this means $\mathbb{E}^{\mathbb{Q}}\left[L\left(T-s_{1}\right) \mid \mathcal{G}\left(T-s_{2}\right)\right]=L\left(T-s_{2}\right)$ for $s_{1}=T-t_{1}, s_{2}=T-t_{2}$, or equivalently:

$$
\mathbb{E}^{\mathbb{Q}}\left[L\left(t_{1}\right) \mid \sigma\left(X(\theta), t_{2} \leq \theta \leq T\right)\right]=L\left(t_{2}\right) .
$$

But this last identity holds for any $T \in\left(t_{2}, \infty\right)$, so it leads - on the strength of the P. LÉVY martingale convergence Theorem 9.4.8 in CHUNG (1974) — to

$$
\mathbb{E}^{\mathbb{Q}}\left[L\left(t_{1}\right) \mid \mathcal{H}\left(t_{2}\right)\right]=L\left(t_{2}\right), \quad \mathcal{H}(t):=\sigma(X(\theta), t \leq \theta<\infty) .
$$

To wit, the likelihood ratio process $(L(t))_{0 \leq t<\infty}$ is a martingale of the backwards filtration $(\mathcal{H}(t))_{0 \leq t<\infty}$, whose "tail" sigma-algebra is trivial on account of the ergodicity property (3.7) of the MARKOV Chain (BLACKWELL \& FREEDMAN (1964)):

$$
\mathcal{H}(\infty):=\bigcap_{0 \leq t<\infty} \mathcal{H}(t)=\{\emptyset, \Omega\}, \quad \bmod . \mathbb{Q} .
$$

We invoke now the martingale version of the backward submartingale convergence Theorem 9.4.7 in Chung (1974). It follows from this result that $(L(t))_{0 \leq t<\infty}$ is a $\mathbb{Q}$-uniformly integrable family; that the limit $L(\infty):=\lim _{t \rightarrow \infty} L(t)$ exists, both a.e. and in $\mathbb{L}^{1}$ under $\mathbb{Q}$; and that the backward martingale property (10.2) extends all the way to infinity, namely

$$
\mathbb{E}^{\mathbb{Q}}\left[L\left(t_{1}\right) \mid \mathcal{H}(\infty)\right]=L(\infty) .
$$

But the triviality under $\mathbb{Q}$ of the tail sigma-algebra, implies that $L(\infty)$ is $\mathbb{Q}$-a.e.constant. Then the extended martingale property (10.3) identifies this constant as $L(\infty)=\mathbb{E}^{\mathbb{Q}}[L(\infty)]=\mathbb{E}^{\mathbb{Q}}\left[L\left(t_{1}\right)\right]=1$.

We recall the relative entropy from (7.5). The convexity of the function $\Phi(\ell)=\ell \log \ell$ shows, in conjunction with (10.2) and the JENSEN inequality, that

$$
(\Phi(L(t)), \mathcal{H}(t))_{0 \leq t<\infty} \quad \text { is a backward } \mathbb{Q}-\text { submartingale, }
$$

with decreasing expectation $\mathbb{E}^{\mathbb{Q}}[\Phi(L(t)]=H(P(t) \mid Q) \geq 0$. Because this expectation is bounded from below, we can appeal once again to the backward submartingale convergence Theorem 9.4.7 in CHUNG (1974). We deduce that the process in (10.4) is a $\mathbb{Q}$-uniformly integrable family which converges, again both a.e. and in $\mathbb{L}^{1}$ under $\mathbb{Q}$, to $\lim _{t \rightarrow \infty} \Phi(L(t))=\Phi(L(\infty))=\Phi(1)=0$.

Furthermore, the aforementioned uniform integrability gives

$$
\lim _{t \rightarrow \infty} \downarrow H(P(t) \mid Q)=\lim _{t \rightarrow \infty} \mathbb{E}^{\mathbb{Q}}[\Phi(L(t))]=\mathbb{E}^{\mathbb{Q}}\left(\lim _{t \rightarrow \infty} \Phi(L(t))\right)=0 ;
$$

that is, (7.9) is also valid in this general case with countable state-space. 


\subsubsection{Relative Entropy is Continuous at the Origin}

We discuss now the validity of the DE BRUIJN identities of (7.18) when the state-space is countable.

Proposition 10.2. The DE BRUIJN identities of (7.18) for the dissipation of relative entropy are valid for a countable state-space, under the finite entropy condition (10.1).

To justify this claim, we would like to use the argument already deployed; but there is now no obvious, general way to turn the local martingale $\widehat{M}^{h}$ of (7.11) into a true $\mathbb{Q}$-martingale. Thus, we localize $\widehat{M}^{h}$ by an increasing sequence $\left\{\sigma_{n}\right\}_{n \in \mathbb{N}}$ of $\widehat{\mathbb{G}}$-stopping-times with values in $[0, T]$ and $\lim _{n \rightarrow \infty} \uparrow \sigma_{n}=T$. In this manner we create the bounded $(\widehat{\mathbb{G}}, \mathbb{Q})$-martingales $\widehat{M}^{h}\left(s \wedge \sigma_{n}\right), 0 \leq s \leq T$, which then give

$$
\begin{aligned}
\mathbb{E}^{\mathbb{Q}} \int_{0}^{\sigma_{n}} & (\partial h+\widehat{\mathcal{K}} h)(u, \widehat{X}(u)) \mathrm{d} u=\mathbb{E}^{\mathbb{Q}}\left[h\left(\sigma_{n}, \widehat{X}\left(\sigma_{n}\right)\right)\right]-\mathbb{E}^{\mathbb{Q}}[h(0, \widehat{X}(0))] \\
\quad & H\left(P\left(T-\sigma_{n}\right) \mid Q\right)-H(P(T) \mid Q) \leq H(P(0) \mid Q)-H(P(T) \mid Q) \leq H(P(0) \mid Q)<\infty
\end{aligned}
$$

for every $n \in \mathbb{N}$, on account of (10.1); see also the argument straddling (10.8) below. In particular, the sequence of real numbers in (10.5) takes values in the compact interval $[-H(P(0) \mid Q), H(P(0) \mid Q)]$.

We would like now to let $n \rightarrow \infty$ in (10.5), and establish the DE BRUIJN identity (7.18) in this case. The issue once again is continuity of the relative entropy — though now at the origin (rather than at infinity, as in (7.9)); and not along fixed times, but rather along an appropriate sequence of stopping times, i.e.,

$$
\lim _{n \rightarrow \infty} \uparrow H\left(P\left(T-\sigma_{n}\right) \mid Q\right)=H(P(0) \mid Q) .
$$

Accepting this for a moment, and letting $n \rightarrow \infty$ in (10.5), we obtain the DE BRUIJN identity (7.18), i.e.,

$$
\int_{0}^{T} I(t) \mathrm{d} t=\mathbb{E}^{\mathbb{Q}} \int_{0}^{T}(\partial h+\widehat{\mathcal{K}} h)(u, \widehat{X}(u)) \mathrm{d} u=H(P(0) \mid Q)-H(P(T) \mid Q)
$$

by monotone convergence. We let now $T \rightarrow \infty$ in (10.7) and arrive at the second identity in (7.18), thanks to the property (7.9) already established in Proposition 10.1.

Proof of (10.6): By analogy with (7.8), and invoking now additionally the optional sampling theorem for the bounded stopping times $\left\{\sigma_{n}\right\}_{n \in \mathbb{N}}$ of $\widehat{\mathbb{G}}$ with values in $[0, T]$, we deduce that the sequence of non-negative real numbers

$$
H\left(P\left(T-\sigma_{n}\right) \mid Q\right)=\mathbb{E}^{\mathbb{Q}}\left[\Phi\left(\ell\left(T-\sigma_{n}, \widehat{X}\left(\sigma_{n}\right)\right)\right)\right], \quad n \in \mathbb{N}
$$

is increasing; in particular, $\lim _{n \rightarrow \infty} H\left(P\left(T-\sigma_{n}\right) \mid Q\right) \leq H(P(0) \mid Q)$. On the other hand, the boundednessfrom-below of the function $\Phi(\ell)=\ell \log \ell$ gives

$$
\lim _{n \rightarrow \infty} H\left(P\left(T-\sigma_{n}\right) \mid Q\right) \geq \mathbb{E}^{\mathbb{Q}}\left[\lim _{n \rightarrow \infty} \Phi\left(\ell\left(T-\sigma_{n}, \widehat{X}\left(\sigma_{n}\right)\right)\right)\right]=\mathbb{E}^{\mathbb{Q}}[\Phi(\ell(0, X(0)))]=H(P(0) \mid Q)
$$

with the help of FATOU's Lemma, and (10.6) follows.

Remark 10.1. The General Case: Exacly the same methods show that the results of Propositions 8.1 and 8.2, pertaining to a general convex function $\Phi:(0, \infty) \rightarrow \mathbb{R}$ with the properties imposed there, continue to hold for the generalized relative entropy functional of (9.2) in the case of a countable state-space $\mathcal{S}$, under the condition $H^{\Phi}(P(0) \mid Q)<\infty$.

Once again, it is important to stress that nowhere in the present Section have we invoked the detailedbalance conditions of (3.11). 


\section{Bibliography}

Ambrosio, L., Gigli, N. \& Savaré, G. (2008) Gradient Flows in Metric Spaces and in the Space of Probability Measures. Second Edition. Lectures in Mathematics, ETH Zürich. Birhäuser Verlag, Basel.

Blackwell, D. \& Freedman, D. (1964) The tail $\sigma$-field of a Markov chain and a theorem of Orey. Annals of Mathematical Statistics 35, 1291-1295.

Bobkov, S.G. \& Tetali, P. (2006) Modified logarithmic Sobolev inequalities in discrete settings. Journal of Theoretical Probability 19, 289-336.

CAputo, P., Dai PrA, P. \& Posta, G. (2009) Convex entropy decay via the Bochner-Bakry-Émery approach. Annales de l' Institut Henri Poincaré (Sér. B, Probabilités et Statistiques) 45, 734-753.

Chafaї, D. (2004) Entropies, convexity and functional inequalities. Journal of Mathematics Kyôto University 42, 325-363.

Chung, K.L. (1974) A Course in Probability Theory. Second Edition, Academic Press, New York.

Conforti, G. (2020) A probabilistic approach to convex $(\phi)$-entropy decay for Markov chains. Preprint, available at https://arxiv.org/abs/2004.10850

Cordero-Erausquin, D. (2002) Some applications of mass transport to Gaussian-type inequalities. Archive for Rational Mechanics and Analysis 161, 257-269.

COURANT, R., Friedrichs, K. \& Lewy, H. (1928) Über die partiellen Differenzellengleichungen der mathematiscen Physik. Mathematische Annalen 100, 32-74.

Cover, T.M. \& Thomas, J.A. (1991) Elements of Information Theory. J. Wiley \& Sons, New York.

Diaconis, P. \& Saloff-Coste, L. (1996) Logarithmic Sobolev inequalities for finite Markov chains. Annals of Applied Probability 6, 695-750.

Dietert, H. (1996) Characterization of gradient flows for finite-state Markov chains. Electronic Communications in Probability 20, no. 29, 1-8.

ERBAR, M. \& MAAS, J. (2012) Ricci curvature of finite Markov Chains via convexity of entropy. Archive for Rational Mechanics and Analysis 206, 997-1038.

ERBAR, M. \& MAAS, J. (2014) Gradient flow structures for discrete porous medium equations. Discrete and Continuous Dynamical Systems 34, 1355-1374.

Fontbona, J. \& Jourdain, B. (2016) A trajectorial interpretation of the dissipations of entropy and Fisher information for stochastic differential equations. Annals of Probability 44, 131-170.

Jordan, R., Kinderlehrer, D. \& OTto, F. (1998) The variational formulation of the Fokker-Planck equation. SIAM Journal of Mathematical Analysis 29, 1-17.

Karatzas, I., Schachermayer, W. \& Tschiderer, B. (2019) Trajectorial Otto Calculus. Preprint (63 pages), preliminary version of [KST 20]. arxiv:1811.08686.

Karatzas, I., Schachermayer, W. \& Tschiderer, B. (2020) A trajectorial approach to the gradient flow properties of Langevin-Smoluchowski diffusions. Condensed version of [KST 19] (37 pages). Submitted, arxiv:2008.09220.

Karatzas, I. \& Shreve, S.E. (1988) Brownian Motion and Stochastic Calculus. Volume 113 of series Graduate Texts in Mathematics. Springer-Verlag, New York. 
Liggett, T.G. (2010) Continuous Time Markov Processes: An Introduction. Volume 113 of the series Graduate Studies in Mathematics. American Mathematical Society, Providence, RI.

MAAS, J. (2011) Gradient flows of the entropy for finite Markov chains. Journal of Functional Analysis 261, 2250-2292.

MAAS, J. (2017) Entropic Ricci curvature for discrete spaces. Lecture Notes in Mathematics 2184, 159173. Springer-Verlag, Berlin.

Miclo, L. (1992) Recuit simulé sans potentiel sur un ensemble fini. Séminaire de Probabilités XXVI, Lecture Notes in Mathematics 1526, 47-60. Springer-Verlag, Berlin

MielKe, A. (2011) A gradient structure for reaction-diffusion systems and for energy-drift-diffusion systems. Nonlinearity 24, 1329-1346.

Mielke, A. (2013) Geodesic convexity of the relative entropy in reversible Markov chains. Calculus of Variations and Partial Differential Equations 48, 1-31.

Mielke, A. (2016) On evolutionary $\Gamma$-convergence for gradient systems. In Macroscopic and Large Scale Phenomena: Coarse Graining, Mean-Field Limits, and Ergodicity. Lecture Notes in Applied Mathematics and Mechanics 3, 187-249. Springer-Verlag, New York.

Montenegro, R. \& Tetali, P. (2006) Mathematical aspects of mixing times in Markov chains. Foundations and Trends in Theoretical Computer Science 1(3), 237-354.

NORRIS, J. (1997) Markov Chains. Cambridge University Press.

Отто, F. (2001) The geometry of dissipative evolution equations: the porous medium equation. Communications in Partial Differential Equations 26, 101-174.

Oтto, F. \& Villani, C. (2000) Generalization of an inequality by Talagrand, and links with the logarithmic Sobolev inequality. Journal of Functional Analysis 173, 361-400.

PAVON, M. (1989) Stochastic control and nonequilibrium thermodynamical systems. Applied Mathematics \& Optimization 19, 187-202.

Rogers, L.C.G. \& Williams, D. (1987) Diffusions, Markov Processes and Martingales. Vol. II: Itô Calculus. J. Wiley \& Sons, New York.

StAm, A.J. (1959) Some inequalities satisfied by the quantities of information of Fisher and Shannon. Information and Control 2, 101-112. 